\title{
Approximate solutions of the incompressible Euler equations with no concentrations
}

by

\author{
Milton C. LOPES FILHO ${ }^{\mathrm{a}, 1}$, Helena J. NUSSENZVEIG LOPES ${ }^{\mathrm{a}, 2}$, \\ Eitan TADMOR ${ }^{\mathrm{b}, 3}$ \\ a Departamento de Matematica, IMECC-UNICAMP, Caixa Postal 6065, \\ Campinas, SP 13081-970, Brasil \\ $\mathrm{b}$ Department of Mathematics, UCLA, Los-Angeles, CA 90095, USA \\ Manuscript received 1 June 1999 \\ To Joel Smoller on his 60th birthday
}

\begin{abstract}
We present a sharp local condition for the lack of concentrations in (and hence the $L^{2}$ convergence of) sequences of approximate solutions to the incompressible Euler equations. We apply this characterization to greatly simplify known existence results for $2 \mathrm{D}$ flows in the full plane (with special emphasis on rearrangement invariant regularity spaces), and obtain new existence results of solutions without energy concentrations in any number of spatial dimensions.

Our results identify the 'critical' regularity which prevents concentrations, regularity which is quantified in terms of Lebesgue, Lorentz, Orlicz and Morrey spaces. Thus, for example, the strong convergence criterion cast in terms of circulation logarithmic decay rates due to DiPerna and Majda is simplified (removing the weak control of the vorticity at infinity) and extended (to any number of space dimensions).
\end{abstract}

Our approach relies on using a generalized div-curl lemma (interesting for its own sake) to replace the role that elliptic regularity theory has

\footnotetext{
${ }^{1}$ E-mail: mlopes@ @ime.unicamp.br.

2 E-mail: hlopes@ime.unicamp.br.

${ }^{3}$ E-mail: tadmor@math.ucla.edu.
} 
played previously in this problem.

(C) 2000 L'Association Publications de l'Institut Henri Poincaré. Published by Elsevier B.V. All rights reserved

AMS classification: $35 \mathrm{Q} 30,76 \mathrm{~B} 03,65 \mathrm{M} 12$

RÉSUMÉ. - On présente une condition locale optimale qui garanti l'inéxistence de concentrations dans des suites de solutions approchées de l'équation d'Euler incompressible (ce qui prouve leur convergence $L^{2}$ ). A l'aide de cette caractérisation on simplifie de façon substantielle les résultats d'existence connus pour les flots 2D dans tout le plan (on insiste tout particulièrement sur les espaces de régularité invariants par réarrangement). On démontre de nouveaux résultats d'existence sans concentrations d'énergie en dimension supérieure.

Notre résultat précise la régularité critique qui empèche l'apparition de concentrations. Cette régularité est quantifiée grâce aux spaces de Lebesgue, Lorentz, Orlicz et Morrey. Ainsi, par exemple, le critère de convergence forte basé dans les termes de circulation logarithmique de corrélations dus à DiPerna et Majda sont simplifiés (en éliminant le contrôle faible de la vorticité à l'infini) et généralisés (en dimension supérieure).

Notre approche est basée sur l'utilisation d'un lemme div-curl généralisé (qui présente un intéret en soi-même) qui remplace le rôle de la régularité elliptique qui était utilisée auparavant.

(C) 2000 L'Association Publications de l'Institut Henri Poincaré. Published by Elsevier B.V. All rights reserved

\section{INTRODUCTION}

Incompressible ideal fluid flow is modeled by the Euler equations:

$$
\left\{\begin{array}{l}
u_{t}+u \cdot \nabla u=-\nabla p \\
\operatorname{div} u=0, \\
\text { initial and boundary data, }
\end{array}\right.
$$

where $u=\left(u_{1}, \ldots, u_{n}\right)$ is the velocity and $p$ the pressure of the flow. This system of equations is physically justified when the effects of viscosity are small. Here, we are particularly interested in irregular flow regimes that attempt to grasp the convective aspects of turbulent flow. It is well known that the ideal flow assumption breaks down at the interface 
between fluids and solids, through boundary layer effects. In general, Eqs. (0.1) may be regarded as an appropriate model for high Reynolds number flow far away from boundaries. Among the initial-boundary value problems one may pose for the system (0.1), the full-space problem is therefore the most natural one.

The theory of weak solutions for Eqs. (0.1) is well developed in two space dimensions. The best results on the existence of weak solutions are existence for initial vorticities in $\mathcal{B M}_{c}^{+} \cap H^{-1}$ due to Delort [19], and for initial vorticities in $L_{c}^{1} \cap H^{-1}$, due to Vecchi and $\mathrm{Wu}$ [44]. One very important open problem is to determine whether these weak solutions conserve kinetic energy or if it is possible to lose energy to the small scales of the flow, i.e., through concentration of energy. Our main concern in this work is to characterize those initial vorticities which generate flows conserving kinetic energy, that is, without concentrations.

Our point of departure is a program set forth by DiPerna and Majda in [16-18] to study the problem of existence of weak solutions. One attempts to prove existence by producing an approximate solution sequence with good a priori estimates and passing to the limit in the weak formulation of the equations. DiPerna and Majda recognized that certain physically interesting 2-D flows (vortex sheet initial data) would naturally give rise to approximate solution sequences that might not converge in $L_{\text {loc }}^{2}$. To deal with that, they introduced the notions of reduced defect measures, concentration sets and concentration dimension, attempting to describe precisely the energy loss in an approximate solution sequence. Their two-pronged approach to the existence problem was to show that the a priori estimates imply that the concentration set is very small, in the sense of Hausdorff dimension, and that if the concentration set is sufficiently small, there exists a weak limit of an approximate solution sequence which is, in fact, a weak solution. This approach was shown to work for stationary problems but has not yet proven useful in the time-dependent problem. Nevertheless, their work has been a very rich source of ideas and originated much of the current research on the weak solutions of the Euler equations.

As part of their investigation, DiPerna and Majda proved two results of particular interest here. The first is an existence result for $2 \mathrm{D}$ flows with initial vorticity in $L_{c}^{p}\left(\mathbb{R}^{2}\right), p>1$ [16, Theorem 2.1]. They obtained a weak solution by showing that the approximate solution sequence generated by mollifying initial data and exactly solving 2DEuler is strongly compact in $L_{\mathrm{loc}}^{2}$. The second result is a criterion for strong convergence of an approximate solution sequence in terms of 
a logarithmic decay condition on the circulation of the flow on small circles, [16, Theorem 3.1]. The subsequent research on this problem has concentrated in determining more precisely for which vorticities in $L^{1}$ one can obtain a strongly compact approximate solution sequence. In $[9,10]$, Chae presented proofs of the existence without concentrations for flows in the full plane with initial vorticities in the Orlicz spaces $L \log L\left(\mathbb{R}^{2}\right)$ and $L(\log L)^{\alpha}\left(\mathbb{R}^{2}\right)$, for $\alpha \geqslant 1 / 2$. In [33] Morgulis solved the problem for flows in a $2 \mathrm{D}$ bounded domain $\Omega$, with initial vorticity in Orlicz spaces contained in $L(\log L)^{1 / 2}(\Omega)$. P.-L. Lions proved an optimal result for bounded domain flows, assuming that the initial vorticity lies in the Lorentz space $L^{(1,2)}(\Omega)$. The results by Morgulis and Lions can be easily extended to periodic flows or flows on a compact manifold.

Our objective here is to propose that the compactness of the sequence of approximate vorticities in $H_{\text {loc }}^{-1}\left(\mathbb{R}^{n}\right)$, which we call $H_{\text {loc }}^{-1}$-stability, is a sharp criterion for the strong convergence of approximate solution sequences for the $n$-dimensional Euler equations. Such an $H_{\mathrm{loc}}^{-1}$-stability condition is implicitly present in Morgulis and Lions' work on the 2D problem. We will see that the sharpness of the $H_{\text {loc }}^{-1}$-stability as a criterion for strong $L^{2}$-compactness is essentially trivial for flows in a bounded domain or on a compact manifold.

We want to put our approach in proper perspective with regard to previous work in this area. To this end, we restrict our attention to two space dimensions and, to further fix ideas, we assume that $\omega^{n}$ is a sequence of approximate vorticities bounded in, say, $L^{p}$, for some $1<$ $p<2$. In rough terms, our problem consists of showing that a bounded, divergence free sequence of vector fields $u^{n}$ in $L^{2}$ is actually precompact by making use of additional information on the $L^{p}$-boundedness of its curl $\omega^{n}=\operatorname{curl} u^{n}$. To pass information from $\omega^{n}$ onto the $u^{n}$ we have to use the ellipticity of the system $\operatorname{div} u^{n}=0$, curl $u^{n}=\omega^{n}$, which has been done in the literature in one of two ways:

1. Study the properties of the Biot-Savart kernel as a singular integral operator, mapping $\omega^{n}$ into $u^{n}$;

2. Introduce the streamfunction $\psi^{n}$, satisfying $\Delta \psi^{n}=\omega^{n}$, and $u^{n}=$ $\nabla^{\perp} \psi^{n}$. Then use well-known regularizing properties of the inverse Laplacian.

The two approaches are equivalent for flows on bounded domains. But these are not equivalent in the full plane.

The first approach in the full plane is based on the Hardy-LittlewoodSobolev Theorem, which together with the $L^{p}$-boundedness of the Riesz transforms imply that the Biot-Savart kernel maps $L^{p}\left(\mathbb{R}^{2}\right)$ into $L^{p^{*}}\left(\mathbb{R}^{2}\right)$ 
continuously. This, together with the Calderon-Zygmund inequality, imply that $u^{n}$ is bounded in $W_{\text {loc }}^{1, p}\left(\mathbb{R}^{2}\right)$, which in turn is compactly imbedded in $L_{\text {loc }}^{2}\left(\mathbb{R}^{2}\right)$.

The boundedness in $W_{\text {loc }}^{1, p}$ of $u^{n}$ cannot be derived, however, using the second approach, because the streamfunction requires an additional a priori bound in $W_{\text {loc }}^{2, p}$. The difficulty lies with the fact that $\psi^{n}$ lacks an a priori $L_{\text {loc }}^{p}$-bound, due to the growth at infinity of the fundamental solution of the Laplacian in 2D (see [35] for an explicit example). ${ }^{4}$ One way of circumventing this difficulty with the second approach, is to have additional control of the vorticity at infinity. This is the role of the hypothesis of weak control of vorticity at infinity, imposed by DiPerna and Majda in their proof of the logarithmic decay of circulation criterion for strong convergence. We note that even with this additional hypothesis, the proof of their result in [16, Theorem 3.1] is extremely laborious. Of course, the first approach is another way to circumvent this difficulty, at the expense of relying on delicate estimates (of singular integrals) that become more intricate to extend to spaces other than $L^{p}$, finally breaking down as we go 'near' $L$ '.

In this work we propose a third approach, in which a (generalized) div-curl lemma plays the role that elliptic regularity theory plays in the first two approaches. With this new approach we recover DiPernaMajda's original $L^{p}$ result, obtain a complete and simplified proof of the $L(\log L)^{\alpha}$ results stated by Chae, extend the $L(\log L)^{\alpha}$ and $L^{(1, q)}$ results by Morgulis and Lions to the full plane, and prove a strengthened version of DiPerna-Majda's logarithmic decay of circulation, greatly simplifying the original proof and eliminating the hypothesis of weak control of vorticity at infinity. Moreover, our approach applies equally well to the general $n$-dimensional case; in particular, we extend the circulation decay criterion (expressed in terms of Morrey regularity) to the $n>2$-dimensional case.

Nothing is said here about uniqueness. In this context we refer the reader to the classical uniqueness results of Yudovich [47] for $\omega(\cdot, t) \in L^{\infty}\left(\mathbb{R}^{n}\right)$, and of Vishik [45] for $\omega(\cdot, t) \in B_{1}^{s}\left(L^{2 / s}\left(\mathbb{R}^{2}\right)\right)$, and

\footnotetext{
${ }^{4}$ This subtle difficulty in implementing the second approach has been overlooked in the proof of [10, Lemma 6], and therefore the proof given by Chae of existence without concentrations for initial vorticities in $L(\log L)^{\alpha}\left(\mathbb{R}^{2}\right), 1 / 2 \leqslant \alpha<1$, is incomplete. We note that an arduous and correct proof of a version of [10, Lemma 6], based on the first approach described above, was given by Schochet in [38].
} 
their recent 'logarithmic' refinements in $[48,46]$. The first examples of nonuniqueness within the class of $L_{\text {loc }}^{2}\left(\mathbb{R}^{n} \times \mathbb{R}\right)$-bounded velocities were constructed by Scheffer [37] and Shnirelman [39].

The remainder of this work is divided as follows. In the first section we introduce the notion of $H_{\mathrm{loc}}^{-1}$-stability and we prove it is a sharp criterion for the strong $L^{2}$-compactness of approximate solution sequences. In Section 2 we obtain existence without concentrations for 2D flows with initial vorticity in $L^{p}, p>1$, in Orlicz spaces contained in $L(\log L)_{c}^{\alpha}$, $\alpha>1 / 2$, and in the Lorentz spaces $L_{c}^{(1, q)}, 1 \leqslant q<2$, by showing that, in each case, the space in question is compactly imbedded in $H_{\mathrm{loc}}^{-1}$. The proof of existence is very simple in these cases. We consider the critical Orlicz space $L(\log L)_{c}^{1 / 2}$ and note that an observation of Chae reduces the problem here to the previous cases. Finally, we present a proof of the $L_{c}^{(1,2)}$ case, based on the ideas of P.-L. Lions for bounded domain flows. We prove that approximate solution sequences corresponding to mollified initial vorticities in $L_{c}^{(1,2)}$ are $H_{\mathrm{loc}}^{-1}$-stable, even though $L_{c}^{(1,2)}$ itself is continuously, but not compactly imbedded in $H_{\text {loc }}^{-1}$. All these 2D cases are singled out as they respect the rearrangement invariance property of the $2 \mathrm{D}$ vorticity. Next, we move beyond the rearrangement invariant case. In Section 3 we prove lack of concentrations if the velocity field belongs to $L^{\infty}\left([0, T] ; L^{p}\left(\mathbb{R}^{2}\right)\right)$ for some $p>2$ with borderline regularity of vorticity in $\mathcal{B M}_{c}\left(\mathbb{R}^{2}\right)$. (The corresponding $n$-dimensional generalization is outlined in Section 4.4). We then turn to the general $n$-dimensional setup. In Section 4 we cast DiPerna-Majda's circulation decay estimates as bounds in the logarithmic Morrey space, $M_{\mathrm{loc}}^{(1 ; \alpha)}\left(\mathbb{R}^{2}\right), \alpha>1$, which we prove to be compactly imbedded in $H_{\mathrm{loc}}^{-1}\left(\mathbb{R}^{2}\right)$. Our argument extends naturally to higher space dimensions where $\widetilde{M}_{c}^{p}\left(\mathbb{R}^{n}\right), p>n / 2$, is compactly imbedded in $H_{\mathrm{loc}}^{-1}\left(\mathbb{R}^{n}\right)$, and the result obtained is related to work on well-posedness of the 3D Navier-Stokes equations with initial vorticity in a Morrey space $\widetilde{M}^{3 / 2}\left(\mathbb{R}^{3}\right)$ due to Giga and Miyakawa [22], and to work by Constantin, E and Titi on the Onsager conjecture [13]. Finally, we conclude with two appendices. In Appendix A we include still another proof which is interesting for its own sake, of the generalized div-curl lemma stated and used in the first section. And in Appendix B we provide a specific example for our convergence results in the context of finite-difference approximations. We present the 2D high-resolution central scheme recently introduced in [27] and we prove its $H_{\text {loc }}^{-1}$-stability. 


\section{STRONG COMPACTNESS OF APPROXIMATE SOLUTIONS- $H_{\mathrm{loc}}^{-1}$-STABILITY}

Let us start by fixing some notations. If $\Phi=\Phi(x)$ is a vector field on $\mathbb{R}^{n}$ then the Jacobian matrix $D \Phi$ has entries $(D \Phi)_{i j}=\partial \Phi^{i} / \partial x_{j}$. A ball of center $x_{0}$ and radius $R$ is denoted $B_{R}\left(x_{0}\right)$. Let $\Omega$ be a smooth domain in $\mathbb{R}^{n}$. If $X$ is any Banach subspace of $\mathcal{D}^{\prime}(\Omega)$ we denote by $X_{c}$ the space of distributions in $X$ with compact support in $\Omega$. We use $W^{k, p}$ to denote Sobolev spaces and $H^{s}$ for the Hilbert spaces $W^{s, 2}$.

We begin by recalling from [16] the definition of approximate solution sequences for the incompressible Euler equations (0.1), defined over any fixed time interval $T>0$.

DEFINITION 1.1 (Approximate Euler solutions). - Let $\left\{u^{\varepsilon}\right\}$ be uniformly bounded in $L^{\infty}\left([0, T] ; L_{\text {loc }}^{2}\left(\mathbb{R}^{n} ; \mathbb{R}^{n}\right)\right)$. The sequence $\left\{u^{\varepsilon}\right\}$ is an approximate solution sequence of the $n$-dimensional incompressible Euler equations (0.1) if the following properties are satisfied.

$\mathcal{P} 1$. The sequence $\left\{u^{\varepsilon}\right\}$ is uniformly bounded in $\operatorname{Lip}\left((0, T) ; H_{1 \mathrm{oc}}^{-L}\left(\mathbb{R}^{n}\right.\right.$; $\left.\left.\mathbb{R}^{n}\right)\right), L>1$.

$\mathcal{P} 2$. For any test vector field $\Phi \in C_{c}^{\infty}\left([0, T) \times \mathbb{R}^{n} ; \mathbb{R}^{n}\right)$ with $\operatorname{div} \Phi=0$ we have:

$$
\begin{aligned}
& \int_{0}^{T} \int_{\mathbb{R}^{n}} \Phi_{t} \cdot u^{\varepsilon}+\left(D \Phi u^{\varepsilon}\right) \cdot u^{\varepsilon} \mathrm{d} x \mathrm{~d} t \\
& \quad+\int_{\mathbb{R}^{n}} \Phi(x, 0) \cdot u^{\varepsilon}(x, 0) \mathrm{d} x \longrightarrow 0 \quad \text { as } \varepsilon \rightarrow 0 .
\end{aligned}
$$

P3. $\operatorname{div} u^{\varepsilon}=0$ in $\mathcal{D}^{\prime}$.

Remarks. -

1. In particular, $u$ is a weak solution of the Euler equations (0.1) if it forms a (fixed) sequence of approximate Euler solutions, $u^{\varepsilon} \equiv u$. Thus, a weak solution, $u$, is an incompressible field, div $u=0$, such that for all test vector fields $\Phi$ 's with $\operatorname{div} \Phi=0$ there holds equality in property $\mathcal{P} 2$,

$$
\int_{0}^{T} \int_{\mathbb{R}^{n}} \Phi_{t} \cdot u+(D \Phi u) \cdot u \mathrm{~d} x \mathrm{~d} t+\int_{\mathbb{R}^{n}} \Phi(x, 0) \cdot u(x, 0) \mathrm{d} x=0 .
$$

2. In the general case, these weak formulations hold in some negative Sobolev space tested against vector fields in $H_{c}^{s}\left([0, T) \times \mathbb{R}^{n} ; \mathbb{R}^{n}\right)$. 
It then follows from the assumed uniform bound on the kinetic energy, $u^{\varepsilon} \in L^{\infty}\left([0, T] ; L_{\text {loc }}^{2}\left(\mathbb{R}^{n} ; \mathbb{R}^{n}\right)\right)$, that $u^{\varepsilon}$ has the Lipschitz regularity required in $\mathcal{P} 1, u^{\varepsilon} \in \operatorname{Lip}\left((0, T) ; H_{\mathrm{loc}}^{-L}\left(\mathbb{R}^{n} ; \mathbb{R}^{n}\right)\right)$, for some $L=L(s, n)>1$, consult [16, Lemma 1]. In particular, any $H^{-s}$ weak solution satisfies property $\mathcal{P} 1$.

3. The results in this paper (following the Main Theorem below) apply to a larger class of approximate solutions $\left\{u^{\varepsilon}\right\}$ than those classified by properties $\mathcal{P} 1-\mathcal{P} 3$. In particular, the requirement of incompressibility $\mathcal{P} 3$ can replaced by the weaker $H^{-1}$-approximate incompressibility, namely

$\mathcal{P} 3^{\prime}$. div $u^{\varepsilon} \rightarrow 0$ in $H_{\text {loc }}^{-1}$.

Let us introduce the curl of a vector field $u=\left(u^{1}, \ldots, u^{n}\right)$ in $\mathbb{R}^{n}$ as the anti-symmetric matrix $\omega=$ curl $u$ whose entries are $\omega_{i j}=\left(u^{i}\right)_{x_{j}}-\left(u^{j}\right)_{x_{i}}$. We will denote the vector space of $n \times n$ anti-symmetric matrices with real entries by $A^{n}$.

DEFINITION $1.2\left(H_{\mathrm{loc}}^{-1}\right.$-stability). - The sequence $\left\{u^{\varepsilon}\right\}$ is called $H_{\mathrm{loc}}^{-1}$ stable if $\left\{\operatorname{curl} u^{\varepsilon}=\omega^{\varepsilon}\right\}$ is a precompact subset of $C\left((0, T) ; H_{\mathrm{loc}}^{-1}\left(\mathbb{R}^{n}\right.\right.$; $\left.\mathbb{A}^{n}\right)$ ).

If $\left\{u^{\varepsilon}\right\}$ is an approximate solution sequence of the $n$-dimensional Euler equations then we will refer to $u^{\varepsilon}$ as velocity and to curl $u^{\varepsilon} \equiv$ $\omega^{\varepsilon}$ as vorticity. Note that, in contrast with [16, Definition 1.1], we have not imposed any conditions on the behavior of $u^{\varepsilon}$ near infinity in Definition 1.1. Consequently, the Biot-Savart Law, which relates vorticity to velocity, is only valid up to an arbitrary harmonic function.

The above definitions are easily adaptable to flows on domains $\Omega$ with boundary; one needs only to remove the "loc" subscript in the function spaces above and add the boundary condition $u^{\varepsilon} \cdot \widehat{n}=0$ on $\partial \Omega$ in the trace sense.

We are now ready to state our main result.

THEOREM 1.1 (Main Theorem). - Let $\left\{u^{\varepsilon}\right\}$ be an approximate solution sequence of the $n$-dimensional Euler equations. If $\left\{u^{\varepsilon}\right\}$ is $H_{\mathrm{loc}}^{-1}$-stable then there exists a subsequence which converges strongly to a weak solution $u$ in $L^{\infty}\left([0, T] ; L_{\text {loc }}^{2}\left(\mathbb{R}^{n} ; \mathbb{R}^{n}\right)\right)$.

The conclusion of the Main Theorem may be referred to as strong compactness of the approximate solution sequence. In particular, $\mathrm{H}_{\mathrm{loc}}^{-1}$ stability is a criterion which excludes the phenomena of concentrations, 
[16]. The proof of this theorem relies heavily on the following timedependent generalization of the classical div-curl lemma of Tartar and Murat [40,34].

LEMMA 1.1 (Generalized div-curl lemma). - Fix $T>0$ and let $\left\{u^{\varepsilon}(\cdot, t)\right\}$ and $\left\{v^{\varepsilon}(\cdot, t)\right\}$ be vector fields on $\mathbb{R}^{n}$ for $0 \leqslant t \leqslant T$. Assume that:

$\mathcal{A} 1 . u^{\varepsilon} \rightarrow u$ and $v^{\varepsilon} \rightarrow v$ weak-* in $L^{\infty}\left([0, T] ; L_{\text {loc }}^{2}\left(\mathbb{R}^{n}\right)\right)$ and strongly in $C\left([0, T] ; H_{\mathrm{loc}}^{-1}\left(\mathbb{R}^{n}\right)\right)$;

A2. $\left\{\operatorname{div} u^{\varepsilon}\right\}$ is precompact in $C\left([0, T] ; H_{\mathrm{loc}}^{-1}\left(\mathbb{R}^{n}\right)\right)$;

$\mathcal{A} 3$. $\left\{\right.$ curl $\left.v^{\varepsilon}\right\}$ is precompact in $C\left([0, T] ; H_{\mathrm{loc}}^{-1}\left(\mathbb{R}^{n} ; \mathbb{A}^{n}\right)\right)$.

Then $u^{\varepsilon} \cdot v^{\varepsilon} \rightarrow u \cdot v$ in $\mathcal{D}^{\prime}\left([0, T] \times \mathbb{R}^{n}\right)$.

This generalization reduces to the classical div-curl lemma (see [40]) when the vector fields $u^{\varepsilon}$ and $v^{\varepsilon}$ are constant in time, since the imbedding $L_{\text {loc }}^{2}\left(\mathbb{R}^{n}\right) \hookrightarrow H_{\text {loc }}^{-1}\left(\mathbb{R}^{n}\right)$ is compact. Although this generalization is not surprising, it has not appeared previously in the literature, and a proofinteresting for its own sake, is given in the appendix. Equipped with the generalized div-curl lemma we now turn to the

Proof of the Main Theorem. - Our objective is to apply the generalized div-curl lemma with the same two vector fields, $v^{\varepsilon}=u^{\varepsilon}$.

Note that since $u^{\varepsilon}$ is divergence-free and $H_{10 c}^{-1}$-stable, hypothesis $\mathcal{A} 2$ and $\mathcal{A} 3$ are automatically satisfied, and thus it remains to verify $\mathcal{A} 1$. Since $\left\{u^{\varepsilon}\right\}$ is assumed to have a uniformly bounded kinetic energy, $u^{\varepsilon} \in L^{\infty}\left([0, T] ; L_{\text {loc }}^{2}\left(\mathbb{R}^{n}\right)\right)$, we can extract a weak-* converging subsequence, $\left\{u^{\varepsilon_{k}}\right\} \rightarrow u$ in $L^{\infty}\left([0, T] ; L_{\text {loc }}^{2}\left(\mathbb{R}^{n}\right)\right.$ ) (for example, by taking a diagonal of weak-* converging subsequences in the $N$-balls, $L^{\infty}\left([0, T] ; L^{2}\left(B_{N}(0)\right)\right.$, and letting $\left.N \rightarrow \infty\right)$. Thus, the first half of $\mathcal{A} 1$ holds. Moreover, the $L_{\text {loc }}^{2}$-energy bound $\left(\left\{u^{\varepsilon}\right\}\right.$ bounded in $\left.L^{\infty}\left([0, T] ; L_{\text {loc }}^{2}\left(\mathbb{R}^{n}\right)\right)\right)$, together with the Lipschitz regularity assumed in $\mathcal{P} 1\left(\left\{u^{\varepsilon}\right\}\right.$ bounded in $\operatorname{Lip}\left((0, T) ; H_{\text {loc }}^{-L}\left(\mathbb{R}^{n} ; \mathbb{R}^{n}\right)\right)$ with $\left.L>1\right)$, produce, by the Aubin-Lions lemma [41], a strongly converging subsequence in $C\left([0, T] ; H_{\mathrm{loc}}^{-1}\left(\mathbb{R}^{n}\right)\right)$, since $L_{\mathrm{loc}}^{2} \stackrel{\text { comp }}{\hookrightarrow} H_{\mathrm{loc}}^{-1} \hookrightarrow H_{\mathrm{loc}}^{-L}$. Thus, the second half of $\mathcal{A} 1$ also holds.

Granted that hypothesis $\mathcal{A} 1-\mathcal{A} 3$ hold we can now apply the generalized div-curl lemma with $u^{\varepsilon}=v^{\varepsilon}$, to conclude that there exists a subsequence $\left\{u^{\varepsilon_{k}}\right\}$ such that $\left|u^{\varepsilon_{k}}\right|^{2} \rightarrow|u|^{2}$ in $\mathcal{D}^{\prime}\left([0, T] \times \mathbb{R}^{n}\right)$. This implies that $u^{\varepsilon_{k}}$ converges strongly to $u$ in $L^{2}\left([0, T] ; L_{\text {loc }}^{2}\left(\mathbb{R}^{n}\right)\right)$. Strong quadratic convergence is all we need: it is then immediate to verify, by passing to the limit in property $\mathcal{P} 2$ of Definition 1.1 , that $u$ is a weak solution. 
Remarks. -

1. We do not know whether the converse of the Main Theorem 1.1 is true. Of course, if $u^{\varepsilon_{k}} \rightarrow u$ strongly in $L^{2}\left([0, T] ; L_{\text {loc }}^{2}\left(\mathbb{R}^{n} ; \mathbb{R}^{n}\right)\right)$ then $\omega^{\varepsilon_{k}} \rightarrow$ curl $u$ strongly in $L^{2}\left([0, T] ; H_{\mathrm{loc}}^{-1}\left(\mathbb{R}^{n} ; \mathbb{A}^{n}\right)\right)$. However, it is not clear how to improve the $L^{2}$ into the $C^{0}$ convergence, required by Definition 1.2 of $H_{\mathrm{loc}}^{-1}$-stability, nor is it clear how a weakened version of that Definition still yields the main Theorem 1.1.

2. In contrast, for flows over bounded simply-connected domains, $H_{\text {loc }}^{-1}$-stability is equivalent to strong compactness. The proof is a trivial consequence of elliptic regularity theory in $H^{-1}$. To see this, fix an approximate solution sequence $\left\{u^{\varepsilon}\right\}$ to the Euler equations on a bounded, smooth simply-connected domain $\Omega \subset \mathbb{R}^{n}$. Denote by $\Delta^{-1}$ the solution operator of the homogeneous Poisson problem, so that $\Delta^{-1}: H^{-1}(\Omega) \rightarrow H_{0}^{1}(\Omega)$.

Since $u^{i, \varepsilon}=\sum_{j=1}^{n} \partial_{x_{j}} \Delta^{-1} \omega_{i j}^{\varepsilon}$, it follows that $\left\{u^{\varepsilon}\right\}$ is precompact in $C\left([0, T] ; L^{2}\left(\Omega ; \mathbb{R}^{n}\right)\right)$ if and only if $\left\{\omega^{\varepsilon}\right\}$ is precompact in $C\left([0, T] ; H^{-1}\left(\Omega ; \mathbb{A}^{n}\right)\right)$.

The interesting consequence of this equivalence is that, if $u^{\varepsilon}$ conserves kinetic energy in time then any strong limit $u$ will as well.

\section{THE 2D PROBLEM-REARRANGEMENT-INVARIANT SPACES IMBEDDED IN $H_{\mathrm{loc}}^{-1}\left(\mathbb{R}^{2}\right)$}

In this section we will concentrate on the initial value problem for the 2-dimensional incompressible Euler equations in the full plane.

The study of incompressible fluid motion in two space dimensions becomes considerably simpler than the $n>2$-dimensional case, because the $2 \mathrm{D}$ vorticity equation reduces to the (scalar) transport equation

$$
\omega_{t}+u \cdot \nabla \omega=0
$$

It is governed by a divergence-free velocity field, $u$, which is recovered by the Biot-Savart law $u=K * \omega$ with $K(\xi):=\xi^{\perp} /\left(2 \pi|\xi|^{2}\right)$. Of course, this is literally true only for smooth solutions, where (2.2) implies that $\left(\partial_{t}+u \cdot \nabla\right) \phi(\omega)=0$ for all smooth $\phi$ 's, and hence, since $u$ is incompressible, that $\omega$ is a renormalized solution, [15], in the sense that

$$
\phi(\omega)_{t}+\nabla \cdot(u \phi(\omega))=0, \quad \forall \phi^{\prime} s
$$


It follows that the total mass, $\int \phi(\omega(\cdot, t))$ is conserved in time, and hence the distribution function of $\omega$ (with respect to the 2D Lebesgue measure), $\lambda_{\omega(\cdot, t)}(\alpha):=\operatorname{meas}\{x|| \omega(x, t) \mid \geqslant \alpha\}$, is also invariant in time. Our task is to carry these arguments of smoothness to the limiting cases of regularity, and to this end one employs appropriate families of (regularized) approximate solutions.

We say that an approximate solution sequence of the 2D incompressible equations, $\left\{u^{\varepsilon}\right\}$, is associated with initial vorticity $\omega_{0}$ if $L_{\text {loc }}^{2}-$ $\lim u^{\varepsilon}(\cdot, 0)=u_{0}=K * \omega_{0}$. Which $\omega_{0}$ give rise to permissible initial velocities $u_{0}=K * \omega_{0} \in L_{\text {loc }}^{2}\left(\mathbb{R}^{2}\right)$ ? Since $K$ belongs to weak- $L^{2}\left(\mathbb{R}^{2}\right)$ and since convolution maps boundedly, $*: L^{2, \infty} \times L^{1, q} \rightarrow L^{2, r \leqslant q}$, it follows that $\omega_{0} \in L^{1,2}$ will do.

Let us mention a few possible approaches to generate approximate solution sequences for the incompressible 2D Euler equations in accordance with Definition 1.1. DiPerna and Majda [16] have indicated that one may obtain approximate solution sequences associated with $\omega_{0} \in \mathcal{B} \mathcal{M}_{c}\left(\mathbb{R}^{2}\right) \cap H^{-1}\left(\mathbb{R}^{2}\right)$ by the following three strategies: ${ }^{5}$

- Mollification of initial data. Using the global well-posedness of the 2D Euler equations for smooth initial data, one obtains a family of approximate solutions, $\left\{u^{\varepsilon}(\cdot, t)\right\}$, corresponding to the mollified initial data, $\left\{u_{0}^{\varepsilon}:=\eta_{\varepsilon} * u_{0}\right\}$ (where $\eta_{\varepsilon}$ denotes any standard Friedrichs mollifier);

- Navier-Stokes solutions. Taking the vanishing viscosity limit of the 2D incompressible Navier-Stokes equations, [41];

- Vortex methods. Approximating the solution by a desingularized vortex (blob) method, [12,2,26]. There is a large literature devoted to the convergence of these methods-consult the recent contribution [29] and the references therein.

In addition, there is a whole variety of classical discrete methods-finitedifference, finite-element and spectral schemes, with particular emphasis on the 2D vorticity formulation. We make no attempt to list them all, but refer instead to prototype studies in [3,7,24,23,21] and the references therein. In particular, in the context of finite difference methods we refer to the high-resolution central scheme recently introduced by Levy and Tadmor in [27], which is singled out in the present context for its notable stability properties. This central scheme and its $H_{\text {loc }}^{-1}$-stability properties are outlined in the appendix.

\footnotetext{
${ }^{5}$ A complete discussion of the relevant temporal estimates involved in Definition 1.1 can be found in [25].
} 
Let $X$ be a rearrangement-invariant Banach function space with respect to Lebesgue measure in $\mathbb{R}^{2}$. Canonical examples are Lebesgue $L^{p}$ spaces, Orlicz $L^{\phi}$ spaces and Lorentz $L^{(p, q)}$ spaces. We refer the reader to [6, Sections 1-2] for a comprehensive discussion. The relevant property of such rearrangement-invariant spaces is that their norm is the same for any pair of (Lebesgue) equimeasurable functions, i.e., $\|f\|_{X}=$ $\|g\|_{X}$ whenever their distribution functions $\lambda_{f}$ and $\lambda_{g}$ coincide. We recall that smooth solutions of the vorticity equation (2.2) form a timeparameterized family, $\omega(\cdot, t)$, of equimeasurable functions, $\lambda_{\omega(\cdot, t)}(\alpha)=$ $\lambda_{\omega_{0}(\cdot)}(\alpha)$. Thus, it is natural, when considering approximate solution sequences $\left\{u^{\varepsilon}\right\}$ associated with $\omega_{0} \in X$, to require the additional estimate:

$$
\left\{\omega^{\varepsilon}\right\} \text { is uniformly bounded in } L^{\infty}([0, T] ; X) .
$$

In fact, since under smooth flows the norm of vorticity in any rearrangement-invariant Banach function space $X$ is a conserved quantity (being a function of $\lambda_{\omega(\cdot, t)}(\alpha)$ ), it is natural to seek approximations that respect the same invariance of $X$-regularity:

$$
\omega_{0}^{\varepsilon} \in X \Rightarrow \omega^{\varepsilon}(t) \in L^{\infty}([0, T], X)
$$

these approximations enjoy the advantage that verifying their initial $X$ regularity, $\left\{\omega_{0}^{\varepsilon}\right\} \in X$, will suffice to guarantee that (2.4) holds at later times. This relation between approximate solutions and rearrangementinvariant spaces is what distinguishes the 2D theory from higher dimensional flows. We turn to a few examples.

- Mollification of initial data. If $X$ is such that the Friedrichs mollifications converge in $X$, then clearly (2.5) holds for approximate solutions, $\left\{u^{\varepsilon}\right\}$ obtained by exactly solving the 2D Euler equations with the mollified initial velocity $u_{0}^{\varepsilon}=K_{\varepsilon} * \omega_{0}$ with $\omega_{0} \in X$, where $K_{\varepsilon}$ denotes the mollified kernel $K_{\varepsilon}:=\eta_{\varepsilon} * K$. This is the generic case which applies to all rearrangement-invariant spaces discussed in this paper: a rearrangement-invariant $X$ is closed under mollification if it contains the continuous functions, $C$, as a dense subset, e.g., [6, Section 3, Lemma 6.1, Lemma 6.3].

- Navier-Stokes approximate solutions. A large class of rearrangement-invariant spaces is provided by Orlicz spaces, $L^{\phi}:=\{f \mid$ $\left.\int \phi(|f(x)|) \mathrm{d} x<\infty\right\}$. With convex $\phi$ 's one has $\varepsilon \phi^{\prime}(\omega) \Delta \omega^{\varepsilon} \leqslant$ $\varepsilon \Delta \phi\left(\omega^{\varepsilon}\right)$ which implies the usual 'entropy' decay in time of $\left\|\omega^{\varepsilon}(\cdot, t)\right\|_{L^{\phi}}$. It follows that (2.5) holds for approximate solutions 
obtained by the vanishing viscosity limit with initial vorticities in Orlicz spaces.

- Vortex blob approximations. There are difficulties in proving (2.4) for vortex blob schemes. See [38] for a thorough discussion of this problem.

- Finite difference schemes. In [27] we introduced a high-resolution central difference approximation of the 2D vorticity equation (2.2) which satisfies the maximum principle. In Appendix B we prove that like the Navier-Stokes approximation, the difference solution, $\omega^{\varepsilon}(\cdot, t)$, maps every Orlicz space $L^{\phi}$ into itself.

Before turning to our main result dealing with rearrangement-invariant spaces $X$, we need to clarify the precise notion of their localized version, $X_{\text {loc }}$. We define $X_{\text {loc }}$ as the Fréchet space determined by the family of seminorms $\|f\|_{k} \equiv\left\|\chi_{B_{k}(0)} f\right\|_{X}$. We say that $X_{\text {loc }}$ is compactly imbedded in $H_{\text {loc }}^{-1}\left(\mathbb{R}^{2}\right)$ if any sequence $\left\{f^{\varepsilon}\right\} \subseteq X_{\text {loc }}$ with each seminorm uniformly bounded, is precompact in $H_{\mathrm{loc}}^{-1}\left(\mathbb{R}^{2}\right)$. We note that this is equivalent to $X\left(B_{k}(0)\right)$ being compactly imbedded in $H^{-1}\left(B_{k}(0)\right)$ for all $k \in \mathbb{N}$.

We are now ready to give the main application of Theorem 1.1 to $2 \mathrm{D}$ flows.

COROLlARY 2.1. - Let $X$ be a rearrangement-invariant Banach space such that $X_{\mathrm{loc}}$ is compactly imbedded in $H_{\mathrm{loc}}^{-1}\left(\mathbb{R}^{2}\right)$. Let $\left\{u^{\varepsilon}\right\}$ be a family of approximate solutions associated with $\omega_{0} \in X$, so that $u^{\varepsilon}(\cdot, 0) \rightarrow u_{0}=K * \omega_{0}$ in $L_{\text {loc }}^{2}$. Assume that $\left\{\omega^{\varepsilon}\right\}$ is uniformly bounded in $L^{\infty}([0, T] ; X)$. Then, $\left\{u^{\varepsilon}\right\}$ is strongly compact in $L^{\infty}\left([0, T] ; L_{\mathrm{loc}}^{2}\left(\mathbb{R}^{2}\right.\right.$; $\left.\mathbb{R}^{2}\right)$ ), and hence it has a strong limit, $u(\cdot, t)$, which is a weak solution associated with $u_{0}$ with no concentrations.

Proof. - Since $\left\{u^{\varepsilon}\right\}$ is a family of approximate solutions in the sense of Definition 1.1 , then property $\mathcal{P} 1$ implies that $\left\{\omega^{\varepsilon}\right\}$ is uniformly bounded in $\operatorname{Lip}\left([0, T] ; H_{\text {loc }}^{-L-1}\left(\mathbb{R}^{2}\right)\right)$. This, together with the assumption that $\left\{\omega^{\varepsilon}\right\}$ is uniformly bounded in $L^{\infty}([0, T] ; X)$ with $X_{\text {loc }} \stackrel{\text { comp }}{\hookrightarrow} H_{\mathrm{loc}}^{-1} \hookrightarrow H_{\mathrm{loc}}^{-L-1}$ imply precompactness in $C\left((0, T) ; H_{\mathrm{loc}}^{-1}\left(\mathbb{R}^{2} ; \mathbb{R}\right)\right)$. Thus $\left\{u^{\varepsilon}\right\}$ is $H_{\mathrm{loc}}^{-1}$ stable and the desired result follows from Theorem 1.1 .

In case of approximate solutions generated by initial mollification we are led to a further simplification which allows us to check only the regularity of the initial vorticity, $\omega_{0}$.

COROLlARY 2.2. - Let $X$ be a rearrangement-invariant Banach space such that $C$ is dense in $X_{\text {loc }}$ which in turn is compactly imbedded in $H_{\mathrm{loc}}^{-1}\left(\mathbb{R}^{2}\right)$. Let $\left\{u^{\varepsilon}\right\}$ be the family of approximate solutions associated 
with the mollified initial vorticity $\omega_{0}^{\varepsilon}:=\eta_{\varepsilon} * \omega_{0} \in X$. Then $\left\{u^{\varepsilon}\right\}$ is strongly compact in $L^{\infty}\left([0, T] ; L_{\text {loc }}^{2}\left(\mathbb{R}^{2} ; \mathbb{R}^{2}\right)\right)$ and hence it has a strong limit, $u(\cdot, t)$, which is a weak solution associated with the initial velocity $u_{0}=K * \omega_{0}$ without concentrations.

The last corollary is particularly useful to identify regularity spaces of initial vorticities which give rise to weak solutions with no concentrations. In the remainder of this section we will use this strategy to derive the existence of concentration-free solutions of the 2D incompressible Euler equations with compactly supported initial vorticities in Lebesgue spaces $L^{p}, p>1$, Orlicz spaces contained in $L(\log L)^{\alpha}, \alpha \geqslant 1 / 2$, and Lorentz spaces $L^{(1, q)}, 1 \leqslant q<2$.

\subsection{Initial vorticity in Lebesgue space $L_{c}^{p}\left(\mathbb{R}^{2}\right), p>1$}

We begin with

THEOREM 2.1. - Let $\omega_{0} \in L_{c}^{p}\left(\mathbb{R}^{2}\right)$ with $p>1$. Then there exists a weak solution of the incompressible 2D Euler equations, $u(\cdot, t)$, associated with the initial conditions $u_{0}=K * \omega_{0}$, with no concentrations.

This result—originally due to Yudovich [47] and DiPerna and Majda [16], follows immediately from Corollary 2.2 , since $L_{\text {loc }}^{p>1}\left(\mathbb{R}^{2}\right)$ is compactly imbedded in $H_{\text {loc }}^{-1}\left(\mathbb{R}^{2}\right.$ ) (and of course, $C_{c}$ is dense in $L^{p<\infty}$ ).

\subsection{Orlicz spaces $L(\log L)_{c}^{\alpha}\left(\mathbb{R}^{2}\right), \alpha \geqslant 1 / 2$}

We now extend our discussion to the more general Orlicz spaces. An Orlicz space, $L^{\phi}$, consists of all measurable functions $f$ such that $\int \phi(|f(x)|) \mathrm{d} x<\infty$. Here, $\phi$ is any admissible $N$-functiona convex function such that $\lim _{s \rightarrow \ell} \phi(s) / s=\ell, \ell \in\{0, \infty\}$. (We refer the interested reader to $[1,6,20]$, for a detailed discussion on Orlicz spaces.) Lebesgue spaces correspond to $\phi(s) \sim s^{p}$, yet Orlicz spaces offer refinement of the Lebesgue ladder of spaces. We consider initial vorticities $\omega_{0}$ in the Orlicz space $L(\log L)^{\alpha}$, corresponding to $\phi(s) \sim$ $s\left(\log ^{+} s\right)^{\alpha}$. Our objective is to simplify and complete the proofs of Chae in $[9,10]$ and extend the results of Morgulis in [33] to full plane flows.

THEOREM 2.2. - Let $\omega_{0} \in L(\log L)_{c}^{\alpha}\left(\mathbb{R}^{2}\right)$ with $\alpha \geqslant 1 / 2$. Then there exists a weak solution of the incompressible 2D Euler equations, $u(\cdot, t)$, subject to the initial condition $u_{0}=K * \omega_{0}$, with no concentrations.

Remark. - We observe that proof of Theorem 2.2 includes any of the admissible Orlicz spaces considered by Morgulis in [33]. 
Proof. - Let $\Omega$ be a bounded smooth domain in $\mathbb{R}^{2}$. We begin by recalling Trudinger's imbedding theorem $[43,20]$, which states that $H_{0}^{1}(\Omega)$ is compactly imbedded in $L^{\psi}(\Omega)$ for any $N$-function $\psi$ which is dominated by $\sigma:=\mathrm{e}^{s^{2}}-1$ ( $\psi$ is dominated by $\sigma$-denoted $\psi \ll \sigma$, if $\lim _{s \rightarrow \infty} \sigma(c s) / \psi(s) \rightarrow \infty$ for all positive $c$ 's). Next we invoke a duality argument. The dual of $L^{\psi}$ is isomorphic to $L^{\phi}$ if the primitives of the corresponding $N$-functions, $\Phi=\int^{s} \phi(r) \mathrm{d} r, \Psi(s)=\int{ }^{s} \psi(r) \mathrm{d} r$, are complementary in the sense that $\Phi \circ \Psi=I d$. Denote $\phi=\widetilde{\psi}$. Then the dual statement of the compact imbedding $H_{0}^{1} \stackrel{\text { comp }}{\hookrightarrow} L^{\psi}$ states that $L^{\phi}$ is compactly imbedded in $H^{-1}(\Omega)$ for any $\phi$ which dominates $\widetilde{\sigma}(s)$, i.e., $\phi \gg s\left(\log ^{+} s\right)^{1 / 2}$.

Let us assume first that $\omega_{0} \in L_{c}^{\phi}\left(\mathbb{R}^{2}\right)$ for some $N$-function $\phi \gg$ $s\left(\log ^{+} s\right)^{1 / 2}$. For example, $\phi_{\alpha}=s\left(\log ^{+} s\right)^{\alpha}$ corresponding to $L(\log L)^{\alpha}$ with $\alpha>1 / 2$. Then Corollary 2.2 applies with the rearrangementinvariant $X \equiv L^{\phi}\left(\mathbb{R}^{2}\right)$ since $X_{\text {loc }} \stackrel{\text { comp }}{\hookrightarrow} H_{\text {loc }}^{-1}$. Hence there exists a subsequence of $u^{\varepsilon}$ which converges strongly in $L_{\text {loc }}^{2}$ to a weak solution, having $u_{0}$ as its initial data.

Finally, we consider the borderline case, $\omega_{0} \in L(\log L)_{c}^{1 / 2}\left(\mathbb{R}^{2}\right)$. Then, as was pointed out by Chae [10], there exists an $N$-function $\phi$ which dominates $\phi_{1 / 2}=s\left(\log ^{+} s\right)^{1 / 2}$ such that $\omega_{0} \in L^{\phi}\left(\mathbb{R}^{2}\right)$, and we conclude as before.

Remark. - We note the special role of $L(\log L)_{c}^{1 / 2}\left(\mathbb{R}^{2}\right)$ which is on the borderline of the logarithmic Orlicz spaces, $L(\log L)^{\alpha}\left(\mathbb{R}^{2}\right), \alpha>$ $1 / 2$, which are compactly imbedded in $H_{\text {loc }}^{-1}\left(\mathbb{R}^{2}\right)$. In Theorem 2.2 we maintain that certain special $L(\log L)_{c}^{1 / 2}$-sequences of approximate vorticities - those corresponding to mollified initial $L(\log L)^{1 / 2}$ vorticities, are $H_{1 \mathrm{loc}}^{-1}$-stable; but, for other sequences, this need not be the case since $L(\log L)_{c}^{1 / 2}\left(\mathbb{R}^{2}\right)$ is not compactly imbedded in $H_{\text {loc }}^{-1}\left(\mathbb{R}^{2}\right)$, as demonstrated by the following

Counterexample. Consider the domain $\Omega=B\left(0 ; \pi^{-1 / 2}\right)$, and the sequence of radial functions, $f_{n}(x)=g_{n}\left(\pi|x|^{2}\right)$ given by

$$
g_{n}(s)= \begin{cases}\frac{\sqrt{\log n}}{\sqrt{2} s(\log s)^{2}}, & 0 \leqslant s \leqslant 1 / n, \\ 0, & 1 / n<s \leqslant 1 .\end{cases}
$$

The function $g_{n}(\cdot)$ is non-negative and non-increasing, provided that $n \geqslant 9$, and thus $f_{n}(\cdot)$ coincides with its decreasing rearrangement, 
$f_{n}(x)=f_{n}^{*}\left(\pi|x|^{2}\right)$. It is easy to estimate, directly from the definition that this sequence is bounded in $L(\log L)^{1 / 2}$. Indeed, expressed in terms of its decreasing rearrangement, the $L(\log L)^{\alpha}(\Omega)$ norm of $f$ (say, with $|\Omega|=1$ ), is given by, e.g. [6, Section 4, Lemma 6.12], $\|f\|_{L(\log L)^{\alpha}}=\int_{0}^{1}(1-\log s)^{\alpha} f^{*}(s) \mathrm{d} s$. A straightforward computation then yields $\left\|f_{n}\right\|_{L(\log L)^{1 / 2}} \sim \sqrt{\log n} \int_{\log n}^{\infty}(1+s)^{-3 / 2} \mathrm{~d} s<$ Const.

Yet, we claim that this sequence is not precompact in $H^{-1}(\Omega)$. To this end we compute the unique solution of $\Delta \psi_{n}=f_{n}$ in $H_{0}^{1}(\Omega)$, which can be done explicitly, using the symmetry of $\Omega$ and of $f_{n}$. It is then a simple calculation that:

$$
\int_{B\left(0 ;(n \pi)^{-1 / 2}\right)}\left|\nabla \psi_{n}\right|^{2}=\frac{1}{8 \pi},
$$

for all $n$. However, $f_{n}$ is precompact in $H^{-1}$ if and only if $\psi_{n}$ is precompact in $H^{1}$, which by the Dunford-Pettis Theorem, implies that $\left\{\left|\nabla \psi_{n}\right|^{2}\right\}$ must be uniformly integrable, which is contradicted by the calculation above.

\subsection{Lorentz spaces $L_{c}^{(1, q)}\left(\mathbb{R}^{2}\right), 1 \leqslant q<2$}

Recall that $\lambda_{f}(\alpha)=$ meas $\{x|| f(x) \mid \geqslant \alpha\}$ denotes the (non-increasing) distribution function of $f$, and let its (generalized) inverse, $f^{*}$, denote the usual non-increasing rearrangement of $f$. The Lorentz spaces $L^{p, q}(\Omega)$ consist of all measurable functions $f$ such that $\int_{0}^{|\Omega|}\left(s^{1 / p} f^{*}(s)\right)^{q} \mathrm{~d} s / s<$ $\infty$. Whereas $L^{p, p}$ coincides with $L^{p}$, Lorentz spaces offer yet another finer grading in the ladder of Lebesgue spaces. We refer the reader to [6] for details. Here we remark that it is customary to use an equivalent characterization of Lorentz spaces where $f^{*}(s)$ is replaced by its maximal function, ${ }^{6} f^{* *}(s):=\frac{1}{s} \int_{0}^{s} f^{*}(r) \mathrm{d} r$. We denote the latter by $L^{(p, q)}$,

$$
L^{(p, q)}:=\left\{f \mid\left\|s^{-1 / p^{\prime}} \int_{0}^{s} f^{*}\right\|_{L^{q}(\mathrm{~d} s / s)}<\infty\right\} .
$$

Whereas $L^{p, q}$ and $L^{(p, q)}$ coincide for $p>1$, they differ, however, for $p=1$, which is precisely the focus of our interest. Indeed, while the

\footnotetext{
${ }^{6} \mathrm{We}$ recall its maximal property to be used later on, namely, $f^{* *}(s)=$ $\sup _{|\Omega|=s} 1 /|\Omega| \int_{\Omega}|f| \mathrm{d} y$.
} 
$L^{1, q}$ 's vary between $L^{1, \infty}=$ weak-[ $\left.L^{1}\right]$ and $L^{1,1}=L^{1}$, the $L^{(1, q)}$ 's are strictly smaller, varying between $L^{(1,1)}=L(\log L)$ and $L^{(1, \infty)}=L^{1}$. The interested reader is referred to a detailed discussion in [4,5]. For the sake of completeness, we outline the following lemma which shows how the Lorentz spaces $L^{(1, q)}$ interlace with the closely related Orlicz spaces we encountered before.

LEMMA 2.1. - The following inclusions hold

$$
L(\log L)^{1 / q}(\Omega) \subset L^{(1, q)}(\Omega) \subset L(\log L)^{\alpha}(\Omega), \quad \alpha<\frac{1}{q} \leqslant 1 .
$$

Proof. - Consider a measurable function $f$, compactly supported on $\Omega$. For simplicity we assume $|\Omega|=1$. The characterization of its $L(\log L)^{\alpha}$-norm is given by (see [6, Section 4 Lemma 6.12]),

$$
\|f\|_{L(\log L)^{\alpha}}=\int_{0}^{1}(1-\log s)^{\alpha} f^{*}(s) \mathrm{d} s .
$$

Integration by parts and the Hölder inequality yield, for $\alpha>0$

$$
\begin{aligned}
\| f & \|_{L(\log L)^{\alpha}} \\
& =\alpha \int_{0}^{1}(1-\log s)^{\alpha-1} \cdot\left(\int_{0}^{s} f^{*}(r) \mathrm{d} r\right) \frac{\mathrm{d} s}{s} \\
& \leqslant \alpha\left(\int_{0}^{1}\left(\int_{0}^{s} f^{*}(r) \mathrm{d} r\right)^{q} \frac{\mathrm{d} s}{s}\right)^{1 / q}\left(\int_{0}^{1}(1-\log s)^{(\alpha-1) q^{\prime}} \frac{\mathrm{d} s}{s}\right)^{1 / q^{\prime}},
\end{aligned}
$$

and hence the estimate $\|f\|_{L(\log L)^{\alpha}} \leqslant$ Const $\left\|\int_{0}^{s} f^{*}\right\|_{L^{q}(\mathrm{~d} s / s)}$ which proves (2.7) for $(1-\alpha) q^{\prime}>1$. The left side of the inclusion in (2.7) as well as a refinement of the inclusion on the right can be found in Lemma 4.1 below.

Using the compact imbedding of Orlicz spaces together with the last lemma yields

THEOREM 2.3. - Let $\omega_{0} \in L_{c}^{(1, q)}\left(\mathbb{R}^{2}\right)$ with $1 \leqslant q<2$. Then there exists a weak solution of the incompressible 2D Euler equations, $u(\cdot, t)$, subject to the initial condition $u_{0}=K * \omega_{0}$ with no concentrations. 


\subsection{The Lorentz space $L_{c}^{(1,2)}\left(\mathbb{R}^{2}\right)$}

The space $L_{c}^{(1,2)}\left(\mathbb{R}^{2}\right)$ will play the role for Lorentz spaces that $L(\log L)_{c}^{1 / 2}\left(\mathbb{R}^{2}\right)$ did for Orlicz spaces. We observe that $L_{c}^{(1,2)}$ is strictly larger than $L(\log L)_{c}^{1 / 2}$ (consult Lemma 2.1). ${ }^{7}$ In fact, it was observed by P.L. Lions that $L^{(1,2)}(\Omega)$ is the largest rearrangement-invariant space which is continuously imbedded in $H^{-1}(\Omega){ }^{8}$

In this subsection we will be concerned with putting together the basic approach of Section 1 and the ideas developed by P.L. Lions for the bounded domain case in [30, Section 4.2] in order to prove existence and absence of concentrations for an initial vorticity $\omega_{0} \in L_{c}^{(1,2)}\left(\mathbb{R}^{2}\right)$.

We first note that a straightforward application of our $H_{\mathrm{loc}}^{-1}$-stability result, e.g., Corollary 2.2 , does not apply, since $L_{c}^{(1,2)}$ is not compactly imbedded in $H_{\text {loc }}^{-1}$; in fact, our counterexample above shows that already its subset $L(\log L)_{c}^{1 / 2}$ lacks $H_{\text {loc }}^{-1}\left(\mathbb{R}^{2}\right)$-compactness. We will show, however, that as in the $L(\log L)_{c}^{1 / 2}$ case, special $L_{c}^{(1,2)}$-sequences of approximate solutions - those corresponding to mollified initial vorticity $\omega_{0}$ with $\omega_{0} \in L_{c}^{(1,2)}\left(\mathbb{R}^{2}\right)$, are $H_{\text {loc }}^{-1}$-stable.

We begin with the following sufficient condition for the $H^{-1}$-precompactness of $L_{c}^{(1,2)}$ subsets, taken from [30, Lemma 4.1].

LEMMA 2.2 (P.L. Lions). - A family $\left\{\omega^{\varepsilon}\right\} \subset L^{(1,2)}(\Omega)$ is precompact in $H^{-1}(\Omega)$ if the following conditions hold:

1. There exists $C>0$ such that $\left\|\omega^{\varepsilon}\right\|_{L^{(1,2)}(\Omega)} \leqslant C$ uniformly in $\varepsilon$,

2. $\int_{0}^{\delta}\left(\int_{0}^{s}\left(\omega^{\varepsilon}\right)^{*}(r) \mathrm{d} r\right)^{2} \frac{\mathrm{d} s}{s} \longrightarrow 0$ as $\delta \rightarrow 0$, uniformly in $\varepsilon$.

Our next step is to prove a basic functional analytic result which will allow us to develop the time-dependent aspect of the work done so far.

Lemma 2.3. - Let $X$ be a reflexive, separable Banach space. Let $\left\{f_{n}\right\}$ be a bounded sequence in $C([0, T] ; X)$. Then $\left\{f_{n}\right\}$ is precompact in $C([0, T] ; X)$ if and only if the following two conditions hold:

(a) $\left\{f_{n}\right\}$ is precompact in $C([0, T] ; w-X)$.

(b) For any $t \in[0, T]$ and for any sequence $t_{n} \rightarrow t$, we have that $\left\{f_{n}\left(t_{n}\right)\right\}$ is precompact in $X$.

\footnotetext{
${ }^{7}$ Let $F(t)$ denote the primitive of $f^{*}$. Then, $f$ belongs to $L(\log L)^{1 / 2}$ if $(\log t)^{-1 / 2} F(t) / t \in L^{1}(0, \delta)$, and it belongs to $L^{(1,2)}$ if $t^{-1 / 2} F(t) \in L^{2}(0, \delta)$. Hence $F(t)=(\log t)^{-1 / 2}(\log \log t)^{-\beta}$ with $1 / 2<\beta<1$ are examples for functions in the gap $L^{(1,2)}-L(\log L)^{1 / 2}$.

${ }^{8}$ We note the difference in notations-Lorentz spaces $L^{(1, q)}$ are denoted as $L^{1, q}$ in [30].
} 
Proof. - It is immediate that if $\left\{f_{n}\right\}$ is precompact in $C([0, T] ; X)$ then conditions (a) and (b) hold.

We now assume (a) and (b). By (a), we can choose a subsequence (without relabeling) such that:

$$
f_{n} \rightarrow f \text { strongly in } C([0, T] ; w-X) .
$$

We will show that this subsequence converges strongly in $C([0, T] ; X)$. Consider $t \in[0, T]$ and a sequence $\left\{t_{n}\right\} \subset[0, T]$ with $t_{n} \rightarrow t$. From the strong convergence in $C([0, T] ; w-X)$ it follows that:

$$
f_{n}\left(t_{n}\right) \rightarrow f(t),
$$

weakly in $X$. Since $\left\{f_{n}\left(t_{n}\right)\right\}$ is strongly precompact, the weak convergence implies that $\left\{f_{n}\left(t_{n}\right)\right\}$ has $f(t)$ as its single possible strong limit. Hence,

$$
f_{n}\left(t_{n}\right) \rightarrow f(t),
$$

in $X$, without needing to pass to a further subsequence.

Our next step is to prove that $f \in C([0, T] ; X)$. It is enough to show that, for any $t \in[0, T]$ and $\left\{t_{n}\right\} \subseteq[0, T]$ with $t_{n} \rightarrow t$, we have that $f\left(t_{n}\right) \rightarrow f(t)$ in $X$. Fix $t$ and $\left\{t_{n}\right\}$ as above. First observe that, for $n$ fixed, $f_{m}\left(t_{n}\right) \rightarrow f\left(t_{n}\right)$, when $m \rightarrow \infty$. Fix $\varepsilon>0$. We choose a subsequence $\left\{f_{m_{n}}\right\}$ such that:

$$
\left\|f_{m_{n}}\left(t_{n}\right)-f\left(t_{n}\right)\right\|_{X}<\varepsilon / 2 .
$$

On the other hand, since $\left\{f_{m_{n}}\right\}$ is a subsequence of $\left\{f_{m}\right\}$ it satisfies (2.8) and condition (b). Therefore we have, as in (2.9):

$$
f_{m_{n}}\left(t_{n}\right) \rightarrow f(t),
$$

as $n \rightarrow \infty$. Hence, there exists $N_{0}=N_{0}(\varepsilon)$ such that, for $n>N_{0}$ :

$$
\left\|f_{m_{n}}\left(t_{n}\right)-f(t)\right\|_{X}<\varepsilon / 2 .
$$

Putting together (2.10) and (2.11), we have shown that $f \in C([0, T] ; X)$.

Now that this is established, it is easy to see that the convergence of $f_{n}$ to $f$ in $C([0, T] ; w-X)$ can be improved to convergence in $C([0, T] ; X)$. This is done by contradiction, using the compactness of $[0, T]$ and (2.9). 
Before we proceed, a couple of remarks are in order.

Remarks. -

1. A compactness derivation of this nature-with $X=L^{2}(\Omega)$ and $f_{n}$ as an approximate velocity sequence for incompressible 2D Euler in a bounded domain $\Omega$ was argued as part of the proof of [30, Theorem 4.3]. Our contribution is to observe that no fluid dynamical considerations are necessary to prove it.

2. The proof of this Lemma shows that, in a very general sense, the absence of sequential concentrations (in the sense of DiPerna and Majda) in a pointwise fashion in time (condition (b)) is equivalent to the absence of dynamical concentrations, i.e., strong temporal continuity in the limit.

We are ready to prove the main result of this subsection, stating an existence result for full plane flows with initial vorticity in $L_{c}^{(1,2)}$, thus extending the corresponding result [30, Theorem 4.3] for flows in bounded domains.

THEOREM 2.4. - Let $\omega_{0} \in L_{c}^{(1,2)}\left(\mathbb{R}^{2}\right)$. Then there exists a weak solution of the incompressible 2D Euler equations, $u(\cdot, t)$ subject to the initial condition $u_{0}=K * \omega_{0}$ with no concentrations.

Proof. - As before we first mollify, denoting by $\omega^{n}(\cdot, t)$ the vorticity corresponding to the mollified initial data, $\omega_{0}^{n}:=\eta_{\varepsilon_{n}} * \omega_{0}$, where $\varepsilon_{n}:=$ $1 / n$. To show $H_{\mathrm{loc}}^{-1}$-stability it is clearly enough to show that for any fixed test function $\varphi \in C_{c}^{\infty}\left(B_{R}(0)\right)$, we have that $\left\{\varphi \omega^{n}\right\}$ is precompact in $C\left([0, T] ; H^{-1}\left(B_{R}(0)\right)\right)$.

We appeal to Lemma 2.3 with $f_{n}=\varphi \omega^{n}$ and $X=H^{-1}\left(B_{R}(0)\right)$. Its requirement (a) holds since $L^{(1,2)}\left(B_{R}(0)\right)$ is boundedly imbbeded in $H^{-1}\left(B_{R}(0)\right)$, and hence, consult [30, Lemma C.1], $\left\{\varphi \omega^{n}\right\}$ is precompact in $C\left([0, T] ; w-H^{-1}\left(B_{R}(0)\right)\right.$.

Next, let $t_{n} \rightarrow t \in[0, T]$, and we turn to consider the second requirement of Lemma 2.3, verifying the required $H^{-1}$-compactness of $\left\{\varphi \omega^{n}\left(\cdot, t^{n}\right)\right\}$. It is an elementary consequence of the definition of the nonincreasing rearrangement functions that $\left(\varphi \omega^{n}(\cdot, t)\right)^{*} \leqslant\|\varphi\|_{L^{\infty}}\left(\omega^{n}(\cdot, t)\right)^{*}$. Furthermore, since $\omega^{n}(\cdot, t)$ is a rearrangement of $\omega_{0}^{n},\left(\omega^{n}(\cdot, t)\right)^{*}=\left(\omega_{0}^{n}\right)^{*}$. And finally,

$$
\int_{0}^{s}\left(\omega_{0}^{n}\right)^{*}(r) \mathrm{d} r=\sup _{|\Omega|=s} \int_{x \in \Omega}\left|\eta_{\varepsilon_{n}} * \omega_{0}(x)\right| \leqslant \int\left|\eta_{\varepsilon_{n}}\right| \cdot \sup _{|\Omega|=s} \int_{x \in \Omega}\left|\omega_{0}(x)\right|
$$




$$
\leqslant \int_{0}^{s}\left(\omega_{0}\right)^{*}(r) \mathrm{d} r
$$

It follows that $f_{n}=\varphi \omega^{n}\left(\cdot, t^{n}\right)$ are uniformly bounded in $L^{(1,2)}$. Moreover,

$$
\int_{0}^{\delta}\left(\int_{0}^{s} f_{n}^{*}(r) \mathrm{d} r\right)^{2} \frac{\mathrm{d} s}{s} \leqslant \int_{0}^{\delta}\left(\int_{0}^{s} \omega_{0}^{*}(r) \mathrm{d} r\right)^{2} \frac{\mathrm{d} s}{s} \rightarrow 0
$$

as $\delta \rightarrow 0$, uniformly in $n$. The precompactness of $\left\{f_{n}\left(\cdot, t^{n}\right)\right\}$ follows from Lemma 2.2.

We can now use Lemma 2.3 with $X=H^{-1}\left(B_{R}(0)\right)$ to conclude the $H_{\text {loc }}^{-1}$-stability. Of course, by Theorem 1.1, we conclude existence of a weak solution for initial vorticities in $L_{c}^{(1,2)}\left(\mathbb{R}^{2}\right)$, without concentrations.

Lemma 2.1 shows that Orlicz space $L(\log L)^{\alpha}(\Omega), \alpha \geqslant 1 / 2$, and Lorentz space $L^{(1, q)}(\Omega), q<2$ (as well as, of course, $L^{p}(\Omega), p>1$ ), are all contained in $L^{(1,2)}(\Omega)$. Hence Theorem 2.4 covers, in fact, all the previous existence results. However, the proof of existence without concentrations in those spaces other than $L^{(1,2)}$ was so much simpler than in $L^{(1,2)}$ that the authors felt justified in including them as independent results. This simplicity will reinforce itself as we turn to the general $n$ dimensional case.

\section{THE 2D PROBLEM-BEYOND REARRANGEMENT-INVARIANT SPACES}

\subsection{Velocity in Lebesgue space $L^{\infty}\left([0, T], L_{\text {loc }}^{p}\left(\mathbb{R}^{2}\right)\right), p>2$}

As the regularity of the $2 \mathrm{D}$ vorticities approaches the limiting case of bounded measures, $\mathcal{B M}_{c}$, (and in fact, even before that-consult the discussion in Section 4.3.1 below on the limiting case of Morrey space $M^{(1 ; 1 / 2)}$, one encounters the phenomenon of energy concentration. Our next theorem shows that if one departs from the borderline case of $L_{\text {loc }}^{2}\left(\mathbb{R}^{2}\right)$ energy bound on the velocity, then there are no concentrations.

THEOREM 3.1. - Let $\left\{u^{\varepsilon}\right\}$ be a family of approximate solutions of the 2D Euler equations (0.1), with vorticities, $\left\{\omega^{\varepsilon}\right\}$, uniformly bounded in $L^{\infty}\left([0, T] ; \mathcal{B} \mathcal{M}_{c}\left(\mathbb{R}^{2}\right)\right)$. Assume that the $\left\{u^{\varepsilon}\right\}$ is uniformly bounded in $L^{\infty}\left([0, T] ; L_{\mathrm{loc}}^{p}\left(\mathbb{R}^{2} ; \mathbb{R}^{2}\right)\right)$ for some $p>2$. Then, $\left\{u^{\varepsilon}\right\}$ is strongly compact 
in $L^{\infty}\left([0, T] ; L_{\text {loc }}^{2}\left(\mathbb{R}^{2} ; \mathbb{R}^{2}\right)\right)$, and hence it has a strong limit, $u(\cdot, t)$, which is a weak solution with no concentrations.

The proof - a straightforward application of the main Theorem 1.1 on $H_{\text {loc }}^{-1}$-stability, proceeds along the lines of the Murat Lemma, [34],[11, Theorem 2.3]. The assumed $L^{p}$ bound on the velocity implies that $\left\{\omega^{\varepsilon}\right\}$ is uniformly bounded in $L^{\infty}\left([0, T] ; X_{p}\right)$ with $X_{p}=W_{\text {loc }}^{-1, p}(\Omega)$ for some $p>2$, and the $\mathcal{B M}_{c}$ bound on the vorticity implies that $\left\{\omega^{\varepsilon}\right\}$ is compactly imbedded in $L^{\infty}\left([0, T] ; X_{q}(\Omega)\right), \forall q<n /(n-$ 1). It follows, by interpolation, that $\left\{\omega^{\varepsilon}\right\}$ is compactly imbedded in $L^{\infty}\left([0, T] ; W_{\mathrm{loc}}^{-1, r}(\Omega)\right)$ for all $r$ 's, $q \leqslant r<p$. This, together with the uniform boundedness of $\left\{\omega^{\varepsilon}\right\}$ in $\operatorname{Lip}\left([0, T] ; H_{\text {loc }}^{-L-1}(\Omega)\right.$ ) (by property $\mathcal{P} 1)$ imply $H_{\text {loc }}^{-1}$-stability and the result follows.

\section{THE GENERAL $n$-DIMENSIONAL PROBLEM}

\subsection{Vorticity in Lebesgue space $L^{\infty}\left([0, T], L_{c}^{p}\left(\mathbb{R}^{n}\right)\right), p>2 n /(n+2)$}

The extension of our $H_{\text {loc }}^{-1}$-stability theory to the general $n$-dimensional case is straightforward. For example, arguing along the lines of Corollary 2.1 we have

THEOREM 4.1. - Let $\left\{u^{\varepsilon}\right\}$ be a family of approximate solutions of the $n$-dimensional Euler equations (0.1), so that properties $\mathcal{P} 1-$ $\mathcal{P} 3$ hold. Assume that $\left\{\omega^{\varepsilon}(\cdot, t)\right\}$ is uniformly bounded in $L^{\infty}([0, T]$, $\left.L_{c}^{p}\left(\mathbb{R}^{n}, \mathbb{A}^{n}\right)\right)$ with $p>\left(p^{*}\right)^{\prime}=2 n /(n+2)$. Then $\left\{u^{\varepsilon}\right\}$ is strongly compact in $L^{\infty}\left([0, T] ; L_{\text {loc }}^{2}\left(\mathbb{R}^{n} ; \mathbb{R}^{n}\right)\right)$, and hence it has a strong limit, $u(\cdot, t)$, which is a weak solution of the Euler equations with no concentrations.

The proof is immediate based on the dual statement of the compact imbedding, $H_{c}^{1}\left(\mathbb{R}^{n}\right) \stackrel{\text { comp }}{\hookrightarrow} L_{c}^{p}\left(\mathbb{R}^{n}\right)$ with $p<p^{*}=2 n /(n-2)$.

Of course, one can refine the above statement with Orlicz and Lorentz scaling. In the general $n$-dimensional case, however, rearrangementinvariant spaces play no special role and therefore we prefer to continue our discussion with the non-rearrangement-invariant Morrey spaces. In order to put the latter in proper perspective, it would be instructive to briefly overview the Lorentz-Zygmund spaces. 


\subsection{Lorentz-Zygmund spaces $L_{c}^{(p, q ; \alpha)}\left(\mathbb{R}^{n}\right)$}

The most refined scaling of the Lebesgue spaces is offered by the Lorentz-Zygmund spaces. We refer the reader to the detailed survey [6, 5]. Here is a readers' digest.

Consider a bounded $\Omega \subset \mathbb{R}^{n}$ (for simplicity, we assume $|\Omega|=1$ ). The space $L^{p, q ; \alpha}=L^{p q}(\log L)^{\alpha}(\Omega)$ consists of all measurable functions $f$ such that $\left\|s^{1 / p}(1-\log s)^{\alpha} f^{*}(s)\right\|_{L^{q}(\mathrm{~d} s / s)}<\infty$. If we replace $f^{*}$ with its maximal function, $f^{* *}$, we obtain the closely related spaces denoted $L^{(p, q ; \alpha)}$; these are the Lorentz-Zygmund spaces which consist of all measurable functions $f$ such that

$$
\|f\|_{L^{(p, q ; \alpha)}(\Omega)}=\left(\int_{0}^{1}\left[s^{1 / p}(1-\log s)^{\alpha} f^{* *}(s)\right]^{q} \frac{\mathrm{d} s}{s}\right)^{1 / q}<\infty, \quad q \leqslant \infty .
$$

Similar to the situation with the regular Lorentz spaces, $L^{(p, q ; \alpha)}$ coincide with $L^{p, q ; \alpha}$ for $p>1$, [5, Corollary 8.2]. For $p=1$, one has, in the notations of [5, Section 11], $L^{(1, q ; \alpha)}=\mathcal{L}^{1 q}(\log \mathcal{L})^{\alpha}$. The secondary index of $q=\infty$ is of particular interest to us. Using the maximality of $f^{* *}(s)=$ $\sup _{|E|=s}(1 /|E|) \int_{E}|f|$, we find that $L^{(p, \infty ; \alpha)}$ consists of all functions $f$ such that

$$
\begin{gathered}
L^{(p, \infty ; \alpha)}=\left\{f\left|\int_{E}\right| f(y) \mid \mathrm{d} y \leqslant \text { Const } \cdot|E|^{1 / p^{\prime}}|1-(\log |E|)|^{-\alpha},\right. \\
\forall|E|<1\} .
\end{gathered}
$$

The $L^{(p, q ; \alpha)}$ 's are rearrangement-invariant spaces which include as special cases both the Lorentz spaces, $L^{(p, q)}=L^{(p, q ; 0)}$, and the logarithmic Orlicz spaces we encountered before, [5, Theorem 11.1],

$$
L(\log L)^{\alpha}(\Omega)=L^{(1,1 ; \alpha-1)}(\Omega) .
$$

The basic hierarchy of these spaces is given by (consult [5, Theorem 9.3], [6, Section 4, Theorem 6.14] for $L^{(p, q ; \alpha)}$ with $p>1$, and [5, Theorem 
11.2] for the corresponding statement for $\left.L^{(1, q ; \alpha)}\right)$ :

$$
L^{(p, a, \alpha)} \subset L^{(p, b, \beta)} \text { if either } \begin{cases}\{\mathrm{i}\} \quad \alpha+1 / a>\beta+1 / b \text { and } \\ (p-1)(a-b) \geqslant 0, \\ \{\text { ii }\} \quad \alpha+1 / a=\beta+1 / b \text { and } a \leqslant b .\end{cases}
$$

We shall concentrate on two cases, depending on whether $n=2$ or $n>2$ :

- The 2D case $(n=2)$. Restricting attention to $p=1$, we find the following refinement of Lemma 2.1.

LEMMA 4.1. - We have the following relations

$$
L(\log L)^{1 / q}(\Omega) \subset L^{(1, q)}(\Omega) \subset L^{(1, \infty ; 1 / q)}(\Omega), \quad \forall q \geqslant 1 .
$$

The left of (4.16) follows from (4.15) with $(a, \alpha, b, \beta)=(1,1 / q-$ $1, q, 0)$. The right of (4.16) (which follows with $(a, \alpha, b, \beta)=$ $(q, 0, \infty, 1 / q))$ offers a refinement of Lemma 2.1, since (4.154.14) imply that $L^{(1, \infty ; 1 / q)} \subset L(\log L)^{\alpha}$ whenever $\alpha q<1$. In fact, the latter can be verified directly in view of the straightforward inequality,

$$
\log (1 / \eta)\left(\int_{0}^{\eta} f^{*}(r) \mathrm{d} r\right)^{q} \leqslant \int_{s=\eta}^{1}\left(\int_{0}^{s} f^{*}(r) \mathrm{d} r\right)^{q} \frac{\mathrm{d} s}{s} \leqslant\|f\|_{L^{(1, q)}}^{q},
$$

which yields the desired estimate for the right side of (4.16)

$$
\int_{E}|f(y)| \mathrm{d} y \leqslant \int_{0}^{|E|} f^{*}(r) \mathrm{d} r \leqslant\|f\|_{L^{(1, q)}}|(\log |E|)|^{-1 / q}, \quad \forall|E|<1 .
$$

- The $n$-dimensional case, $n>2$. Here we restrict attention to $\alpha=0$, where $L^{(p, q ; 0)}$ coincide with the usual Lorentz spaces, $L^{(p, q ; 0)}=$ $L^{p, q}$ for $p>1$.

\subsection{Morrey spaces $M_{c}^{(p ; \alpha)}\left(\mathbb{R}^{n}\right)$ and $\widetilde{M}_{c}^{(p ; \alpha)}\left(\mathbb{R}^{n}\right)$}

In this section we will consider vorticities in multidimensional Morrey spaces. These further extend the Lorentz-type spaces we encountered so far. 
Motivated by the Lorentz-Zygmund spaces $L^{(p, \infty ; \alpha)}$, we introduce the Morrey-type spaces $M^{(p ; \alpha)}(\Omega)$ which consist of all $L_{\text {loc }}^{1}(\Omega)$-functions such that

$$
\begin{aligned}
\|f\|_{M^{(p ; \alpha)}(\Omega)}:= & \sup _{R>0}\left[R^{-n / p^{\prime}}\left(1-(\log R)_{-}\right)^{\alpha} \sup _{x \in \mathbb{R}^{n}} \int_{B_{R}(x) \cap \Omega}|f| \mathrm{d} y\right]<\infty, \\
& \Omega \subset \mathbb{R}^{n} .
\end{aligned}
$$

(Of course, one might further refine this definition with a secondary index $q$ along the lines of $\left.L^{(p, q ; \alpha)}\right)$. A comparison with (4.13) shows that these Morrey spaces enlarge the ladder of spaces beyond the LorentzZygmund spaces

$$
L^{(p, \infty ; \alpha)}(\Omega) \subset M^{(p ; \alpha)}(\Omega) .
$$

We shall concentrate on two cases depending whether $n=2$ or $n>2$.

In two space dimensions we will consider the logarithmic Morrey spaces $M^{(1 ; \alpha)}(\Omega) \supset L^{(1, \infty ; \alpha)}(\Omega)$

$$
M^{(1 ; \alpha)}(\Omega):=\left\{f\left|\sup _{x \in \mathbb{R}^{2}} \int_{B_{R}(x) \cap \Omega}\right| f \mid \mathrm{d} y \leqslant C\left(1-(\log R)_{-}\right)^{-\alpha}, \forall R>0\right\} .
$$

We conclude that the ladder of two-dimensional spaces is endowed with a complete hierarchy, $L(\log L)^{\alpha}(\Omega) \subset L^{(1,1 / \alpha)}(\Omega) \subset L^{(1, \infty ; \alpha)}(\Omega) \subset$ $M^{(1 ; \alpha)}(\Omega)$.

In the $n$-dimensional case, $n>2$, we consider the canonical Morrey space, $M^{(p ; 0)}(\Omega)=M^{p}(\Omega)$ for $p>1$,

$$
M^{p}(\Omega):=\left\{f\left|\sup _{x \in \mathbb{R}^{n}} \int_{B_{R}(x) \cap \Omega}\right| f \mid \mathrm{d} y \leqslant C R^{n / p^{\prime}}, \forall R>0\right\} .
$$

In the present context, we inquire about the compact imbedding of these space in $H_{\mathrm{loc}}^{-1}\left(\mathbb{R}^{n}\right)$. This issue is addressed in the following theorem, proved independently by DeVore and Tao. ${ }^{9}$

TheOREM 4.2 (R. DeVore; T. Tao). - Let $\Omega \subset \mathbb{R}^{n}$ be a bounded domain and let $\left\{\omega^{\varepsilon}\right\} \subset C_{c}^{\infty}(\Omega)$ be a bounded sequence in $M^{(p ; \alpha)} \cap$ $L^{1}(\Omega)$. If either:

(a) $p>n / 2$, or,

\footnotetext{
${ }^{9}$ Private communication.
} 
(b) $p=n / 2$ and $\alpha>1$,

then $\left\{\omega^{\varepsilon}\right\}$ is precompact in $H^{-1}\left(\mathbb{R}^{n}\right)$.

Proof. - For simplicity, assume that $\Omega$ is included within the unit $n$-box, $Q_{0}=[0,1]^{n}$. We will consider an orthonormal wavelet basis for $L^{2}(\Omega),\left\{\psi_{j k}\right\}$. This basis may be built using a (finite) wavelet set, $\Psi=\{\psi\}$, supported in $Q_{0}$, which we will require to belong to $H^{1}\left(\mathbb{R}^{n}\right)$ (consult [14, Section 3.6] for a brief overview). Specifically, the wavelet basis consists of

$$
\psi_{j k}(x):=2^{k n / 2} \psi\left(2^{k} x-j\right), \quad k \in \mathbb{Z}^{+}, j \in \mathbb{Z}^{n}, \psi \in \Psi,
$$

which are supported in the dyadic cubes $Q_{j k}:=2^{-k}\left(Q_{0}+j\right)$; of course, $\operatorname{diam}\left(Q_{j k}\right) \sim 2^{-k}$ for all $j$ 's.

Next, we consider the wavelet expansion of each $\omega^{\varepsilon}$ :

$$
\omega^{\varepsilon}=\sum_{\psi \in \Psi} \sum_{k \in \mathbb{Z}^{+}} \sum_{j \in \mathbb{Z}^{n}} \omega_{j k}^{\varepsilon} \psi_{j k}, \quad \omega_{j k}^{\varepsilon}=\int_{Q_{j k}} \omega^{\varepsilon} \psi_{j k} \mathrm{~d} x .
$$

The boundedness of $\left\{\omega^{\varepsilon}\right\}$ in Morrey's $M^{(p: \alpha)}$ yields two type of bounds on the corresponding wavelet coefficients. First, each $Q_{j k}$ has a volume of $2^{-k n}$ and is covered by a ball with radius $R=c 2^{-k}$, and hence

$$
\left|\omega_{j k}^{\varepsilon}\right| \leqslant 2^{k n / 2} \int_{Q_{j k}}\left|\omega^{\varepsilon}\right| \leqslant \text { Const } \cdot\left\|\omega^{\varepsilon}\right\|_{M^{(p ; \alpha)}} \cdot 2^{k n / 2} 2^{-k n / p^{\prime}}\left|1+k_{+}\right|^{-\alpha} .
$$

Next, we estimate the $\ell^{1}\left(\mathbb{Z}^{n}\right)$-norm of the wavelet coefficients $\left\{\omega_{j k}^{\varepsilon}\right\}_{j \in Z^{n}}$ in terms of the $\left\|\omega^{\varepsilon}\right\|_{L^{1}}$. In fact, since for each fix $k$, the $Q_{j k}$ 's form a pairwise disjoint cover of $\Omega$, we have

$$
\sum_{j \in \mathbb{Z}^{n}}\left|\omega_{j k}^{\varepsilon}\right| \leqslant \text { Const } \cdot 2^{k n / 2} \sum_{j \in \mathbb{Z}^{n}} \int_{Q_{j k}}\left|\omega^{\varepsilon}\right| \leqslant \text { Const } \cdot 2^{k n / 2}\left\|\omega^{\varepsilon}\right\|_{L^{1}} .
$$

We conclude that the $\omega^{\varepsilon}$ 's are bounded in $H^{-1}$. Indeed, the $\psi_{j k}$ 's are $H^{-1}$-orthogonal, each of which does not exceed $\left\|\psi_{j k}\right\|_{H^{-1}}^{2} \leqslant$ $\min \left\{2^{-2 k} \int|\widehat{\Psi}(\eta)|^{2} /|\eta|^{2}, 1\right\}$, and hence

$$
\left\|\omega^{\varepsilon}\right\|_{H^{-1}}^{2}=\sum_{\psi \in \Psi} \sum_{(j, k) \in\left(\mathbb{Z}^{n}, \mathbb{Z}^{+}\right)}\left|\omega_{j k}^{\varepsilon}\right|^{2}\left\|\psi_{j k}\right\|_{H^{-1}}^{2} \leqslant \text { Const } \sum_{k \in \mathbb{Z}^{+}} 2^{-2 k} \sum_{j \in \mathbb{Z}^{n}}\left|\omega_{j k}^{\varepsilon}\right|^{2} .
$$


Using $(4.21) \times(4.22)$ we find the upper-bound

$$
\begin{aligned}
\left\|\omega^{\varepsilon}\right\|_{H^{-1}}^{2} & \leqslant \text { Const } \sum_{k \in \mathbb{Z}^{+}} 2^{-2 k} \cdot \sup _{j}\left|\omega_{j k}^{\varepsilon}\right| \times\left(\sum_{j \in \mathbb{Z}^{n}}\left|\omega_{j k}^{\varepsilon}\right|\right) \\
& \leqslant \text { Const } \sum_{k \in \mathbb{Z}^{+}} 2^{k(n / p-2)}\left|1+k_{+}\right|^{-\alpha}
\end{aligned}
$$

which shows that $\omega^{\varepsilon}$ are $H^{-1}$-bounded if either (a) or (b) holds. Moreover, we have $H^{-1}$-compactness of $\left\{\omega^{\varepsilon}\right\}$ in view of the uniform summability

$$
\begin{aligned}
& \left\|\sum_{k>k_{0}} \sum_{j \in \mathbb{Z}^{n}} \omega_{j k}^{\varepsilon} \psi_{j k}\right\|_{H^{-1}} \\
& \quad \leqslant \text { Const } \sum_{k>k_{0}} 2^{k(n / p-2)}\left|1+k_{+}\right|^{-\alpha} \rightarrow 0, \quad \text { uniformly in } \varepsilon .(4.23)
\end{aligned}
$$

The uniform high-frequency decay (in $H^{-1}$ ) converts weak compactness in $H^{-1}$ into a strong one.

Next, following [22], we introduce the spaces $\widetilde{M}^{(p ; \alpha)}$ as the spaces of measures $\mu \in \mathcal{B} \mathcal{M}_{\text {loc }}$ with the estimates analogous to (4.17)

$$
\|\mu\|_{\widetilde{M}^{(p ; \alpha)}}:=\sup _{R>0}\left[R^{-n / p^{\prime}}\left(1-(\log R)_{-}\right)^{\alpha} \sup _{x \in \mathbb{R}^{n}}|\mu|\left(B_{R}(x)\right)\right]<\infty .
$$

Our objective is to extend Theorem 4.2 to a result regarding the compact imbedding of $\widetilde{M}^{(p ; \alpha)}$ in $H^{-1}\left(\mathbb{R}^{n}\right)$. Unfortunately, these spaces are not separable and we can not perform the obvious density argument suggested by Theorem 4.2. We will work around this difficulty with an argument inspired on Lemma 2.3.

THEOREM 4.3. - Let $\Omega \subset \mathbb{R}^{n}$ be a bounded domain. Then $\widetilde{M}^{p ; \alpha} \cap$ $\mathcal{B M}(\Omega)$ is compactly imbedded in $H^{-1}(\Omega)$ if $(p-n / 2)_{+}+(\alpha-1)_{+}>$ 0 .

Proof. - We denote by $X$ either $\widetilde{M}^{(1, \alpha)}(\Omega)$, if $\Omega \subseteq \mathbb{R}^{2}$ with some $\alpha>1$ or $\widetilde{M}^{p}(\Omega)$, if $\Omega \subseteq \mathbb{R}^{n}, n \geqslant 3$, with some $p>n / 2$.

Given $\mu \in X$, define $\mu^{m} \equiv \mu * \eta^{m}$, where $\eta^{m}$ is a standard Friedrichs mollifier. It is easy to see that $\mu^{m}$ may not converge to $\mu$ in the topology of $X$. Our first observation is that the $X$-norms of $\mu^{m}$ do converge to the $X$-norm of $\mu$ nevertheless (this property is, in a sense, inherited from $\mathcal{B} \mathcal{M}$, where it is rather trivial). Indeed, the total variation (which is the 
norm of $\mu$ in $\mathcal{B M}$ ) is lower semicontinuous with respect to the weak-* convergence in $\mathcal{B} \mathcal{M}$. Hence, by the definition of the Morrey norms:

$$
\|\mu\|_{X} \leqslant \liminf _{m}\left\|\mu^{m}\right\|_{X} .
$$

On the other hand, the space $\mathcal{B M}$ is a Banach algebra under convolution, so that, once again using the definition of the Morrey norms we get:

$$
\left\|\mu^{m}\right\|_{X} \leqslant\left\|\eta^{m}\right\|_{\mathcal{B} \mathcal{M}}\|\mu\|_{X}=\|\mu\|_{X} .
$$

Consequently,

$$
\left\|\mu^{m}\right\|_{X} \rightarrow\|\mu\|_{X} \quad \text { as } m \rightarrow \infty .
$$

Next we use (4.24) and the proof of Theorem 4.2 to deduce that $X$ is continuously imbedded in $H^{-1}$. Let $\varphi \in C_{c}^{\infty}(\Omega)$. Then:

$$
\begin{aligned}
\langle\mu, \varphi\rangle & =\lim _{m \rightarrow \infty}\left\langle\mu^{m}, \varphi\right\rangle \leqslant \liminf _{m \rightarrow \infty}\left\|\mu^{m}\right\|_{H^{-1}}\|\varphi\|_{H^{1}} \\
& \leqslant \liminf _{m \rightarrow \infty} C\left\|\mu^{m}\right\|_{X}\|\varphi\|_{H^{1}}=C\|\mu\|_{X}\|\varphi\|_{H^{1}},
\end{aligned}
$$

where, in the last inequality, we used (the proof of) Theorem 4.2. We conclude that $X$ is in fact continuously imbedded in $H^{-1}$.

We will now prove the compactness of the imbedding. Let $\left\{\mu^{i}\right\}$ be a bounded sequence in $X$. By what we have just proved, we may extract a subsequence $\left\{\mu^{i_{k}}\right\}$, converging weakly in $H^{-1}$ to $\mu \in H^{-1}$. We will have concluded the proof once we show that $\mu^{i_{k}}$ in fact converges strongly to $\mu$.

We introduce the mollifications $\mu^{i, m} \equiv \eta^{m} * \mu^{i}$ and observe that, since $\left\{\mu^{i}\right\}$ is uniformly bounded in $H^{-1}$, the mollifications $\mu^{i, m}$ converge weakly in $H^{-1}$ to $\mu^{i}$ as $m \rightarrow \infty$, uniformly in $i$. Indeed, for $\varphi \in H_{0}^{1}$ :

$$
\left|\left\langle\mu^{i, m}-\mu^{i}, \varphi\right\rangle\right|=\left|\left\langle\mu^{i}, \eta^{m} * \varphi-\varphi\right\rangle\right| \leqslant C\left\|\eta^{m} * \varphi-\varphi\right\|_{H^{1}},
$$

which converges to zero as $m \rightarrow \infty$, uniformly in $i$.

From the fact that $\mu^{i_{k}}$ is bounded in $X$ and (4.24), we have that, for any increasing sequence of natural numbers $\left\{m_{k}\right\}$, the sequence of mollifications $\left\{\mu^{i_{k}, m_{k}}\right\}$ is bounded in $X$ and, by Theorem 4.2, is precompact in $H^{-1}$. Because $\mu^{i_{k}}$ is weakly convergent, we can prove that $\left\{\mu^{i_{k}, m_{k}}\right\}$ is not only strongly compact, but actually strongly convergent to the weak limit $\mu$ of $\mu^{i_{k}}$. To see that, we consider some subsequence of $\mu^{i_{k}, m_{k}}$ (which, for obvious reasons, we do not relabel), strongly convergent in $H^{-1}$ to some $v$. Then: 


$$
\begin{aligned}
0 & =\lim _{k \rightarrow \infty}\left\langle\mu^{i_{k}, m_{k}}, \varphi\right\rangle-\langle v, \varphi\rangle \\
& =\lim _{k \rightarrow \infty}\left\langle\mu^{i_{k}, m_{k}}-\mu^{i_{k}}, \varphi\right\rangle+\left\langle\mu^{i_{k}}, \varphi\right\rangle-\langle v, \varphi\rangle \\
& =\langle\mu, \varphi\rangle-\langle v, \varphi\rangle,
\end{aligned}
$$

since

$$
\left|\lim _{k \rightarrow \infty}\left\langle\mu^{i_{k}, m_{k}}-\mu^{i_{k}}, \varphi\right\rangle\right| \leqslant C \lim _{k \rightarrow \infty}\left\|\eta^{m_{k}} * \varphi-\varphi\right\|_{H^{1}}=0 .
$$

Hence $\mu=v$. Since the converging subsequence above was arbitrary, we may conclude that $\mu^{i_{k}, m_{k}}$ converges strongly to $\mu$ in $H^{-1}$ as we wished.

Note that, by standard properties of mollifications, we have that for each fixed $i, \mu^{i, m} \rightarrow \mu^{i}$ strongly in $H^{-1}$ as $m \rightarrow \infty$.

We are now ready to conclude the proof, by proving that $\mu^{i_{k}}$ converges strongly to $\mu$. Let $\varepsilon>0$ and choose $m_{k}$ an increasing sequence of natural numbers such that:

$$
\left\|\mu^{i_{k}, m_{k}}-\mu^{i_{k}}\right\|_{H^{-1}}<\varepsilon / 2
$$

Also choose $K$ sufficiently large so that if $k>K$ then:

$$
\left\|\mu^{i_{k}, m_{k}}-\mu\right\|_{H^{-1}}<\varepsilon / 2 .
$$

Consequently, $\mu^{i_{k}} \rightarrow \mu$ strongly in $H^{-1}$.

We now return to incompressible flows, beginning with the 2-dimensional case.

\subsubsection{Vorticity in 2D Morrey space $\widetilde{M}_{c}^{(1 ; \alpha)}\left(\mathbb{R}^{2}\right), \alpha>1$}

We have

THEOREM 4.4. - Let $\left\{u^{\varepsilon}\right\}$ be a family of approximate solutions of the 2D Euler equations (0.1), and assume that the corresponding sequence of vorticities $\left\{\omega^{\varepsilon}\right\}$ is uniformly bounded in $L^{\infty}\left([0, T] ; \widetilde{M}_{c}^{(1 ; \alpha)}\right.$ $\left.\left(\mathbb{R}^{2}\right)\right)$, with $\alpha>1$. Then, $\left\{u^{\varepsilon}\right\}$ is strongly compact in $L^{\infty}\left([0, T] ; L_{\text {loc }}^{2}\left(\mathbb{R}^{2}\right.\right.$; $\left.\mathbb{R}^{2}\right)$ ), and has a strong limit, $u(\cdot, t)$, which is a weak solution with no concentrations.

The proof, based on the $H_{\mathrm{loc}}^{-1}$-stability asserted in Theorem 4.3, follows along the lines of Corollary 2.1. 
Remarks. -

1. The result stated in Theorem 4.4 is originally due to DiPerna and Majda [16, Theorem 3.1]. They show, using elliptic regularity, that the streamfunction corresponding to approximate vorticity $\omega^{\varepsilon}(\cdot, t) \in \widetilde{M}_{c}^{(1 ; \alpha)}\left(\mathbb{R}^{2}\right)$ has a logarithmic Hölder regularity of order $\alpha-1$,

$$
\left|\psi^{\varepsilon}(x)-\psi^{\varepsilon}(y)\right| \leqslant \text { Const } \cdot|\log | x-y||^{1-\alpha}, \quad \alpha>1,|x-y|<1 / 2 \text {. }
$$

Here we extend their result in both generality and simplicity. In particular, we remove their extra assumption of weak uniform control at infinity on the approximate vorticities, since our approach based on the generalized div-curl lemma is local, in contrast to the elliptic regularity used in [16, Theorem 3.1].

2. Why Morrey spaces? bounds in Morrey spaces have physical significance as estimates on the decay rates of circulation of the flow. Since $M^{(1 ; \alpha)}\left(\mathbb{R}^{2}\right)$ are not rearrangement-invariant spaces, however, there is no known strategy for obtaining a priori Morreytype bounds. One interesting exception that was observed by Majda, [31], deals with approximate vorticities, $\left\{\omega^{\varepsilon}\right\}$ in the cone of positive measures in $H^{-1}\left(\mathbb{R}^{2}\right)$ : conservation of the pseudoenergy, $-\int \psi^{\varepsilon} \omega^{\varepsilon}(\cdot, t)$, implies that $\omega^{\varepsilon} \in \mathcal{B} \mathcal{M}_{c}^{+}$are contained in $\widetilde{M}_{c}^{(1 ; 1 / 2)}\left(\mathbb{R}^{2}\right)$. In [31], Majda has shown how the Morrey regularity in $\widetilde{M}_{c}^{(1 ; 1 / 2)}\left(\mathbb{R}^{2}\right)$ of one-signed vorticities, plays a fundamental role in his simplified proof of the concentration-cancelation argument of Delort [19]. We note in passing that in fact, the concentrationcancelation property is guaranteed by Morrey regularity, though with no a priori specified rate. That is, if $\sup _{x} \int_{B_{R}(x)}\left|\omega^{\varepsilon}(x)\right| \mathrm{d} x \leqslant$ $\phi(R) \underset{R \rightarrow 0}{\rightarrow} 0$, then the corresponding $L_{\text {loc }}^{2}\left(\mathbb{R}^{2}\right)$ velocity field satisfies the concentration-cancelation property, $u_{i}^{\varepsilon} \cdot u_{j}^{\varepsilon} \rightarrow \bar{u}_{i} \cdot \bar{u}_{j}$ for $i \neq j$.

3. The discussion above leaves us with a gap between those weak solutions obtained from approximate solution sequences without concentrations, corresponding to $\alpha>1$, and approximate solutions with concentration associated, for example, with positive vorticities corresponding to $\alpha=1 / 2$ (for a specific example, consult [16, Section 1]). The gap $1 / 2<\alpha<1$ remains open with regard to the question of compact imbedding of $\widetilde{M}_{c}^{(1 ; \alpha)}\left(\mathbb{R}^{2}\right)$ in $H_{\mathrm{loc}}^{-1}\left(\mathbb{R}^{2}\right)$. 
4.3.2. Vorticity in $n$-dimensional Morrey space $\widetilde{M}_{c}^{p}\left(\mathbb{R}^{n}\right), p>n / 2$

We now consider three and higher dimensional flows. First we remark that the Morrey space estimates do not have the physical interpretation as circulation decay estimates in higher dimensions. On the other hand, there is no natural set of a priori estimates for vorticity, in contrast with dimension two. We state

THEOREM 4.5. - Let $\left\{u^{\varepsilon}\right\}$ be a family of approximate solutions of the $n$-dimensional Euler equations (0.1), and assume that the corresponding sequence of vorticities $\left\{\omega^{\varepsilon}\right\}$ is uniformly bounded in $L^{\infty}\left([0, T] ;\left(\widetilde{M}_{\mathrm{loc}}^{p}\right)\right.$ $\left.\left(\mathbb{R}^{n}\right)\right)$, with $p>n / 2$. Then, $\left\{u^{\varepsilon}\right\}$ is strongly compact in $L^{\infty}([0, T]$; $\left.L_{\text {loc }}^{2}\left(\mathbb{R}^{n} ; \mathbb{R}^{n}\right)\right)$, and hence it has a strong limit, $u(\cdot, t)$, which is a weak solution with no concentrations.

Remarks. -

1. In the particular case of three-space dimensions, Theorem (4.5) indicates that the Morrey space $\widetilde{M}^{3 / 2}\left(\mathbb{R}^{3}\right)$ is the borderline case for $H^{-1}$-compactness and hence existence without concentrations. This space was already addressed by Giga and Miyakawa [22] who proved existence and uniqueness of a global solution to the 3D incompressible Navier-Stokes (N-S) equations on the full space with initial vorticity sufficiently small in $\widetilde{M}^{3 / 2}\left(\mathbb{R}^{3}\right)$.

2. Why Morrey spaces $\widetilde{M}^{n / 2}\left(\mathbb{R}^{n}\right)$ with $n>2$ ? One important feature of the work [22] is that it includes vortex ring initial data, i.e., initial vorticities supported on closed curves. These vorticities generate physically interesting flows that do not have locally finite kinetic energy. In particular, generalizing this example in the obvious way for higher dimensions, this implies that the condition $p>n / 2$ is optimal for our compactness results inside the Morrey hierarchy, since we can exhibit compactly supported measures in $\widetilde{M}^{n / 2}\left(\mathbb{R}^{n}\right)$ which are not in $H_{\mathrm{loc}}^{-1}\left(\mathbb{R}^{n}\right)$, for $n \geqslant 3$.

We note in passing that Morrey regularity is also related to the sharp partial regularity result for the 3D Navier-Stokes equations obtained by Caffarelli, Kohn and Nirenberg [8]; consult also the simplified proofs in $[28,42]$. These results involve an a priori spacetime Morrey-type estimate of the form

$$
\sup _{0<R<R_{0}} \frac{1}{R} \int_{B_{R}(x, t)}\left|\omega^{\varepsilon}(y, s)\right|^{2} \mathrm{~d} y \mathrm{~d} s \leqslant \text { Const } \ll 1,
$$


where $B_{R}(x, t)$ denotes the space-time parabolic ball, $B_{R}(x, t):=$ $B_{R}(x) \times\left(t-R^{2}, t\right)$. We observe that this kind of bound respects the basic similarity invariance of $\mathrm{N}-\mathrm{S}$ solutions, $w_{\lambda}(x, t):=$ $\lambda^{2} w\left(\lambda x, \lambda^{2} t\right), \forall \lambda>0$. Our arguments are essentially stationary and cannot be directly interpreted in this context. Under the assumptions of [42, Theorem 3.1] one concludes $\sup _{B_{R / 2}(x, t)}|\nabla u| \leqslant$ Const $\cdot R^{-2}$ and (4.25) then yields Morrey boundedness in $M^{3 / 2}\left(\mathbb{R}^{3}\right)$ we encountered before.

3. And finally, we comment on the existing gap between Morrey vs. Lebesgue regularity. Theorem 4.1 implies that within the Lebesgue hierarchy, the critical Lebesgue exponent is $\left(p^{*}\right)^{\prime}=2 n /(n+2)$, so that all $L_{c}^{p, \infty}\left(\mathbb{R}^{n}\right)$ with $p>2 n /(n+2)$ are compactly imbedded in $H_{\text {loc }}^{-1}\left(\mathbb{R}^{n}\right)$. Theorem 4.2 , however, identifies the critical Morrey spaces $M_{c}^{p}\left(\mathbb{R}^{n}\right)$ for a smaller range of exponents with $p>n / 2$. Though the Morrey space are bigger than the corresponding weak$L^{p}, L^{p, \infty} \subset M^{p}$, they both admit the same scaling. Thus, for $n>2$ we are left with the open question with regard to the 'correct' scaling exponent within the intermediate gap $n / 2>p>$ $2 n /(n+2)$, which will suffice for compact imbedding in $H_{\text {loc }}^{-1}\left(\mathbb{R}^{n}\right)$.

\subsection{Velocity in Lebesgue space $L^{\infty}\left([0, T], L_{\mathrm{loc}}^{p}\left(\mathbb{R}^{n}\right)\right), p>2$}

The space $L_{c}^{\frac{2 n}{n+2}}\left(\mathbb{R}^{n}\right)$ is the borderline case within the Lebesgue hierarchy for the regularity (lack of concentration) of approximate $n$ dimensional vorticities, analogous to the borderline role of $L_{c}^{1}\left(\right.$ or $\left.\mathcal{B} \mathcal{M}_{c}\right)$ in the 2D setup. Observe, for example, that with $K(\xi) \sim|\xi|^{1-n} \in L^{\frac{n}{n-1}}, \infty$ one finds for the corresponding velocity field, $K * \omega: L_{c}^{2 n /(n+2)}\left(\mathbb{R}^{n}\right) \longmapsto$ $L_{\text {loc }}^{2}\left(\mathbb{R}^{n}\right)$.

Following the 2D setup of Theorem 3.1 we have

THEOREM 4.6. - Let $\left\{u^{\varepsilon}\right\}$ be a family of approximate solutions of the $n$-dimensional Euler equations (0.1), with vorticities, $\left\{\omega^{\varepsilon}\right\}$, uniformly bounded in $L^{\infty}\left([0, T] ; L_{c}^{\frac{2 n}{n+2}}\left(\mathbb{R}^{n}\right)\right)$. Assume that the $\left\{u^{\varepsilon}\right\}$ is uniformly bounded in $L^{\infty}\left([0, T] ; L_{\mathrm{loc}}^{p}\left(\mathbb{R}^{n} ; \mathbb{R}^{n}\right)\right)$ with $p>2$. Then, $\left\{u^{\varepsilon}\right\}$ is strongly compact in $L^{\infty}\left([0, T] ; L_{\text {loc }}^{2}\left(\mathbb{R}^{n} ; \mathbb{R}^{n}\right)\right)$, and hence it has a strong limit, $u(\cdot, t)$, which is a weak solution with no concentrations.

For the proof one makes use of the interpolation argument between $X_{r}$ 's, $X_{r}=W^{-1, r}\left(\mathbb{R}^{n}\right)$. The compact imbedding $L_{c}^{2 n /(n+2)}\left(\mathbb{R}^{n}\right) \stackrel{\text { comp }}{\hookrightarrow} X_{q}$ 
with $q<2$ together with the boundedness of $\left\{\omega^{\varepsilon}\right\}$ in $X_{p}$ for some $p>2$ imply $H^{-1}$ stability.

Here, there are no known strategy to guarantee the $L^{r}$ bounds on the vorticity (for $r \geqslant 2 n /(n+2)$ ) and the velocity (for $r>2$ ).

\section{CONCLUDING REMARKS}

The analysis developed in this paper is related to the question of characterizing weak solutions which conserve kinetic energy. This question was considered for 3D-flow by Onsager in [36], in connection with conditions of asymptotic validity for Kolmogorov's description of the energy spectrum of turbulent flow in the inertial range. More recently, P. Constantin, W. E and E. Titi, [13] proved that weak solutions of the 3D incompressible Euler equations for periodic flow conserve kinetic energy if the velocity belongs to $L^{3}\left([0, T] ; B_{\infty}^{s}\left(L^{3}\left(\mathbb{R}^{3}\right)\right)\right) \cap C\left([0, T] ; L^{2}\left(\mathbb{R}^{3}\right)\right)$, for any $s>1 / 3$, answering in the affirmative part of the Onsager conjecture, that the energy would be conserved if and only if the velocity is at least Hölder continuous of exponent $1 / 3$. We observe that our work shows that flows which are much more irregular may still conserve kinetic energy. For instance, consider a periodic, or bounded domain flow obtained as a limit of an approximate solution sequence of velocities in $L^{\infty}\left([0, T] ; W^{s, p}\right)$, for any $s>(3 / p)-(3 / 2)$. Then, in view of the discussion in the end of Section 1, this flow conserves kinetic energy. Finally, note that this observation does not disprove the "only if" part of the Onsager conjecture, since we are not able to produce such an approximate solution sequence. We are merely saying that it is conceivable that such a counterexample exists.

\section{ACKNOWLEDGEMENT}

Research supported in part by CNPq grant \#300.962/91-6 and FAPESP grant \#96/7635-4 (M.C. L-F), CNPq grant \#300.158/93-9 (H.J. N-L), and ONR grant N00014-91-J-1076 and NSF grant \#DMS97-06827 (E.T). H. and M. Lopes thank Indiana Univ. Math. Dept. for their hospitality. E. Tadmor is grateful to Ron DeVore, Pierre-Louis Lions and Terry Tao for stimulating discussions, and to Bobby Sharpley for pointing out the key references $[4,5]$. 


\section{APPENDIX A. A GENERALIZED DIV-CURL LEMMA}

Below we present a proof of Lemma, 1.1. This proof is based on a new proof of the classical div-curl lemma, [40,34].

Proof. - The first (and standard) step is to observe that, without loss of generality, one can assume $\left\{u^{\varepsilon}\right\}$ and $\left\{v^{\varepsilon}\right\}$ share a single compact support and the same weak limit $u=v=0$.

Indeed, fix $\varphi \in C_{c}^{\infty}\left([0, T] \times \mathbb{R}^{n}\right)$ and let $\psi \in C_{c}^{\infty}\left([0, T] \times \mathbb{R}^{n}\right)$ be such that $\psi \equiv 1$ on the support of $\varphi$. Let $\sqcup^{\varepsilon}=\varphi\left(u^{\varepsilon}-u\right)$ and $\vee^{\varepsilon}=\psi\left(v^{\varepsilon}-v\right)$. It is easy to see, passing to a subsequence if necessary, that $\sqcup^{\varepsilon} \rightarrow 0$ and $\vee^{\varepsilon} \rightarrow 0$ weak-* in $L^{\infty}\left([0, T] ; L_{\text {loc }}^{2}\left(\mathbb{R}^{n}\right)\right)$ and strongly in $C\left([0, T] ; H_{\mathrm{loc}}^{-1}\left(\mathbb{R}^{n}\right)\right)$, so that assumption $\mathcal{A} 1$ holds. Moreover, $\left\{\operatorname{div} \sqcup^{\varepsilon}\right\}$ and $\left\{\operatorname{curl} \vee^{\varepsilon}\right\}$ are precompact in $C\left([0, T] ; H_{\text {loc }}^{-1}\left(\mathbb{R}^{n}\right)\right)$ and respectively in $C\left([0, T] ; H_{\mathrm{loc}}^{-1}\left(\mathbb{R}^{n} ; \mathbb{A}^{n}\right)\right.$ ) (and thus assumptions $\mathcal{A} 2-\mathcal{A} 3$ hold). Indeed, from the hypothesis, the only non-trivial condition to verify is that $\operatorname{div}(\varphi u)$ and $\operatorname{curl}(\psi v)$ belong to $C\left([0, T] ; H^{-1}\left(\mathbb{R}^{n}\right)\right)$ and to $C\left([0, T] ; H^{-1}\left(\mathbb{R}^{n} ; \mathbb{A}^{n}\right)\right)$, respectively. This follows since, passing to a subsequence if necessary, $\operatorname{div}\left(\varphi u^{\varepsilon}\right)$ converges strongly in $C\left([0, T] ; H^{-1}\left(\mathbb{R}^{n}\right)\right)$ to a limit which has to equal $\operatorname{div}(\varphi u)$ a.e. in time; analogously for $\operatorname{curl}(\psi v)$.

Our next step is at the heart of matter. Denote

$$
r^{\varepsilon}:=\operatorname{div} \sqcup^{\varepsilon}, \quad S^{\varepsilon}:=\operatorname{curl} \vee^{\varepsilon}, \quad a^{\varepsilon}:=\widehat{\sqcup}^{\varepsilon}, \quad b^{\varepsilon}:=\widehat{\nabla^{\varepsilon}} .
$$

Fourier transform in the spatial variables leads to the following system

$$
\left\{\begin{array}{l}
\widehat{r^{\varepsilon}}=i a^{\varepsilon} \cdot \xi \\
\widehat{S^{\varepsilon}}=i\left(b^{\varepsilon} \otimes \xi-\xi \otimes b^{\varepsilon}\right) .
\end{array}\right.
$$

We view these as a system of $(1 / 2) n(n-1)+1$ equations in the $n$ unknowns $\xi$. In the 2 -dimensional case, one finds a $2 \times 2$ system which is easily inverted; in the $n$-dimensional case, $n>2$, it is an over-determined system. In either case, we can solve this elliptic system and recover $\xi$ as long as the inner product of $a^{\varepsilon}$ and $b^{\varepsilon}$ does not vanish. To this end, multiply the second equation on the right by $\overline{a^{\varepsilon}}$ and use the first equation to obtain $\left\langle b^{\varepsilon}, a^{\varepsilon}\right\rangle \xi=i\left(\widehat{S^{\varepsilon}} \overline{a^{\varepsilon}}+\overline{r^{\varepsilon}} b^{\varepsilon}\right)$. Take the inner product of both sides of the last equality by the vector $\xi /|\xi|^{2}$. We obtain our main identity which reads

$$
\left\langle b^{\varepsilon}, a^{\varepsilon}\right\rangle \equiv i\left(\frac{\left\langle\widehat{S^{\varepsilon}} \overline{a^{\varepsilon}}, \xi\right\rangle}{|\xi| \cdot|\xi|}+\frac{\overline{r^{\varepsilon}}}{|\xi|} \frac{\left\langle b^{\varepsilon}, \xi\right\rangle}{|\xi|}\right),
$$

where 


$$
\left\{\begin{array}{l}
\left(a^{\varepsilon}, b^{\varepsilon}\right):=\left(\widehat{\sqcup^{\varepsilon}}, \widehat{\mathrm{V}^{\varepsilon}}\right) \\
\left(r^{\varepsilon}, S^{\varepsilon}\right):=\left(\operatorname{div} \sqcup^{\varepsilon}, \operatorname{curl} \vee^{\varepsilon}\right) .
\end{array}\right.
$$

Thus, we can integrate (A.1) on $[0, T] \times \mathbb{R}^{n}$ and use the Plancherel Theorem and the Cauchy-Schwartz inequality to get:

$$
\begin{aligned}
\int_{0}^{T} \int_{\mathbb{R}^{n}} \sqcup^{\varepsilon} \cdot \vee^{\varepsilon} \mathrm{d} x \mathrm{~d} t \leqslant & \left(\int_{0}^{T} \int_{\mathbb{R}^{n}} \frac{\left|\widehat{r^{\varepsilon}}\right|^{2}}{|\xi|^{2}} \mathrm{~d} \xi \mathrm{d} t\right)^{1 / 2}\left\|\vee^{\varepsilon}\right\|_{L^{2}\left([0, T] ; L^{2}\left(\mathbb{R}^{n}\right)\right)} \\
& +\left(\int_{0}^{T} \int_{\mathbb{R}^{n}} \frac{\left\|\widehat{S^{\varepsilon}}\right\|^{2}}{|\xi|^{2}} \mathrm{~d} \xi \mathrm{d} t\right)^{1 / 2}\left\|\sqcup^{\varepsilon}\right\|_{L^{2}\left([0, T] ; L^{2}\left(\mathbb{R}^{n}\right)\right)}
\end{aligned}
$$

Therefore, to conclude that $\int_{0}^{T} \int_{\mathbb{R}^{n}} \sqcup^{\varepsilon} \cdot \vee^{\varepsilon} \mathrm{d} x \mathrm{~d} t \rightarrow 0$, it is enough to show that both $\widehat{r^{\varepsilon}} /|\xi|$ and $\widehat{S^{\varepsilon}} /|\xi|$ converge to 0 strongly in $L^{2}([0, T] \times$ $\mathbb{R}^{n}$ ). We will give the proof for $r^{\varepsilon}$; the proof for $S^{\varepsilon}$ is analogous.

We proceed by separating the low and high Fourier modes:

$$
\begin{aligned}
\int_{0}^{T} \int_{\mathbb{R}^{n}} \frac{\left|\widehat{r^{\varepsilon}}\right|^{2}}{|\xi|^{2}} \mathrm{~d} \xi \mathrm{d} t & =\int_{0}^{T} \int_{|\xi| \leqslant 1} \frac{\left|\widehat{r^{\varepsilon}}\right|^{2}}{|\xi|^{2}} \mathrm{~d} \xi \mathrm{d} t+\int_{0}^{T} \int_{|\xi|>1} \frac{\left|\widehat{r^{\varepsilon}}\right|^{2}}{|\xi|^{2}} \mathrm{~d} \xi \mathrm{d} t \\
& =: \mathcal{I}_{1}+\mathcal{I}_{2} .
\end{aligned}
$$

The assumption of $H^{-1}$ compactness tells us precisely that the high Fourier modes vanish in the limit $\varepsilon \rightarrow 0$ :

$$
\mathcal{I}_{2} \leqslant 2 \int_{0}^{T} \int_{|\xi|>1} \frac{\left|\widehat{r^{\varepsilon}}\right|^{2}}{|\xi|^{2}+1} \mathrm{~d} \xi \mathrm{d} t \leqslant 2 \int_{0}^{T}\left\|r^{\varepsilon}\right\|_{H^{-1}}^{2} \mathrm{~d} t \rightarrow 0 .
$$

Of course, the low-modes are already controlled by the weak convergence (to zero), since $\widehat{r^{\varepsilon}}(\xi, t)=\xi \cdot \widehat{\sqcup^{\varepsilon}}(\xi, t)$, and hence $\left|\widehat{r^{\varepsilon}}(\xi, t)\right|^{2} /|\xi|^{2} \leqslant$ $\left|\widehat{\sqcup}^{\varepsilon}(\xi, t)\right|^{2}$. But the $\widehat{\sqcup^{\varepsilon}}$ are uniformly bounded in $L^{\infty}\left([0, T] \times \mathbb{R}^{n}\right)$ (since the $\sqcup^{\varepsilon}$ are in $\left.L^{\infty}\left([0, T] ; L^{1}\left(\mathbb{R}^{n}\right)\right)\right)$, and they converge pointwise to zero: since $\sqcup^{\varepsilon} \rightarrow 0$ in $C\left([0, T] ; H^{-1}\left(\mathbb{R}^{n}\right)\right)$ and

$$
\begin{gathered}
\widehat{\sqcup^{\varepsilon}}(\xi, t)=\int_{\mathbb{R}^{n}} \mathrm{e}^{-i x \cdot \xi} \psi(x, t) \sqcup^{\varepsilon}(x, t) \mathrm{d} x \rightarrow 0, \\
\mathrm{e}^{-i x \cdot \xi} \psi(x, t) \in C\left([0, T] ; H_{0}^{1}\left(\mathbb{R}^{n}\right)\right) .
\end{gathered}
$$

Consequently, by dominated convergence, $\int_{0}^{T} \int_{|\xi|<1}\left|\widehat{\cup^{\varepsilon}}\right|^{2}$, and hence $\mathcal{I}_{1}$, tends to zero. 
From the fact that $\int_{0}^{T} \int_{\mathbb{R}^{n}} \sqcup^{\varepsilon} \cdot \vee^{\varepsilon} \mathrm{d} x \mathrm{~d} t \rightarrow 0$ it follows that, at least up to subsequence,

$$
\int_{0}^{T} \int_{\mathbb{R}^{n}} \varphi u^{\varepsilon} \cdot v^{\varepsilon} \mathrm{d} x \mathrm{~d} t \rightarrow \int_{0}^{T} \int_{\mathbb{R}^{n}} \varphi u \cdot v \mathrm{~d} x \mathrm{~d} t .
$$

To prove that the entire sequence $\int_{0}^{T} \int_{\mathbb{R}^{n}} \varphi u^{\varepsilon} \cdot v^{\varepsilon} \mathrm{d} x \mathrm{~d} t \rightarrow \int_{0}^{T} \int_{\mathbb{R}^{n}} \varphi u$. $v \mathrm{~d} x \mathrm{~d} t$ we observe that this is the only possible limit of any converging subsequence of the bounded sequence of real numbers $\left\{\int_{0}^{T} \int_{\mathbb{R}^{n}} \varphi u^{\varepsilon}\right.$. $\left.v^{\varepsilon} \mathrm{d} x \mathrm{~d} t\right\}$. This concludes the proof.

\section{APPENDIX B. A HIGH-RESOLUTION CENTRAL SCHEME AND ITS $H_{\mathrm{loc}}^{-1}$-STABILITY}

We describe the approximation of $(0.1)$ by the central scheme recently introduced in [27]. It enjoys the advantages of having high-resolution while avoiding spurious oscillations. Indeed, we extended the maximum principle studied in [27, Theorem 4.1], proving the more general $\mathrm{H}_{\mathrm{loc}}^{-1}$ stability statement below.

The solution of this central scheme is realized as a piecewise-constant approximate solution, $\omega(x, y, t)$, at the discrete time levels, $t^{n}=n \Delta t$, $\omega\left(x, y, t^{n}\right)=\sum_{j, k} \bar{\omega}_{j, k}^{n} 1_{\mathcal{C}_{j, k}}$, with cell-averages, $\bar{\omega}_{j, k}^{n}$, corresponding to the cells, $\mathcal{C}_{j, k}:=\left\{(\xi, \zeta)|| \xi-x_{j}|\leqslant \Delta x / 2,| \zeta-y_{k} \mid \leqslant \Delta y / 2\right\}$.

To evolve in time, we first need to be able to recover the velocity field $(u, v)$ from the discrete vorticity. To this end, we define the discrete vorticity at the mid-cells as the average of the four corners of each cell, i.e.

$$
\bar{\omega}_{j+\frac{1}{2}, k+\frac{1}{2}}:=\frac{1}{4}\left(\bar{\omega}_{j+1, k+1}+\bar{\omega}_{j, k+1}+\bar{\omega}_{j, k}+\bar{\omega}_{j+1, k}\right) .
$$

We then use a streamfunction, $\psi$, such that $\Delta \psi=-\bar{\omega}$, which is obtained in the mid-cells, e.g., by solving the five-points Laplacian,

$$
\Delta \psi_{j+\frac{1}{2}, k+\frac{1}{2}}=-\bar{\omega}_{j+\frac{1}{2}, k+\frac{1}{2}} .
$$

Then, its gradient, $\nabla \psi$ recovers the velocity field

$$
u_{j, k}:=\mu_{x} \nabla_{y} \psi_{j, k}, \quad v_{j, k}:=-\mu_{y} \nabla_{x} \psi_{j, k}, \quad\left(\nabla_{x}^{2}+\nabla_{y}^{2}\right) \psi=-\bar{\omega} .
$$


Here and below, $\mu_{x}, \mu_{y}$ denote averaging in the $x$ - and $y$-directions, e.g., $\mu_{x} f_{\alpha}:=\frac{1}{2}\left(f_{\alpha+1 / 2}+f_{\alpha-1 / 2}\right)$, and similarly, $\nabla_{x}, \nabla_{y}$ denote $x$ - and $y$-differencing, e.g., $\nabla_{y} g_{\beta}:=\frac{1}{\Delta y}\left(g_{\beta+1 / 2}-g_{\beta-1 / 2}\right)$. Thus, for example, (B.2) amounts to

$$
u_{j, k}=\frac{1}{2 \Delta y}\left(\psi_{j+\frac{1}{2}, k+\frac{1}{2}}-\psi_{j+\frac{1}{2}, k-\frac{1}{2}}+\psi_{j-\frac{1}{2}, k+\frac{1}{2}}-\psi_{j-\frac{1}{2}, k-\frac{1}{2}}\right) .
$$

Observe that with this integer indexed velocity field, we retain the following discrete incompressibility relation (centered around $(j+1 / 2, k+$ $1 / 2)$ ),

$$
\mu_{y} \nabla_{x} u_{j+\frac{1}{2}, k+\frac{1}{2}}+\mu_{x} \nabla_{y} v_{j+\frac{1}{2}, k+\frac{1}{2}}=0 .
$$

This discrete incompressibility plays the key role in the $H^{-1}$ stability study below.

Equipped with the velocity values at $t^{n}$ we advance in time in two steps: we first compute the midvalues, $\omega_{j, k}^{n+1 / 2}$, using a first-order Taylor expansion $\left(\lambda_{x}:=\Delta t / \Delta x\right.$ and $\lambda_{y}:=\Delta t / \Delta y$, are the usual fixed meshratios),

$$
\omega_{j, k}^{n+\frac{1}{2}}=\bar{\omega}_{j, k}^{n}-\frac{\lambda_{x}}{2}(u \omega)_{j, k}^{\prime}-\frac{\lambda_{y}}{2}(v \omega)_{j, k}^{\prime},
$$

and using these midvalues, the vorticity at the next time step $t^{n+1}=$ $t^{n}+\Delta t$ is then realized by its staggered cell-averages,

$$
\omega\left(x, y, t^{n+1}\right)=\sum_{j, k} \bar{\omega}_{j+\frac{1}{2}, k+\frac{1}{2}}^{n+1} 1_{\mathcal{C}_{j+\frac{1}{2}, k+\frac{1}{2}}},
$$

where

$$
\begin{aligned}
\bar{\omega}_{j+\frac{1}{2}, k+\frac{1}{2}}^{n+1}= & \mu_{x} \mu_{y} \bar{\omega}_{j+\frac{1}{2}, k+\frac{1}{2}}^{n}+\frac{1}{8} \mu_{y} \nabla_{x} \omega_{j+\frac{1}{2}, k+\frac{1}{2}}^{\prime}+\frac{1}{8} \mu_{x} \nabla_{y} \omega_{j+\frac{1}{2}, k+\frac{1}{2}}^{\prime} \\
& -\Delta t\left\{\mu_{y} \nabla_{x}(u \omega)_{j+\frac{1}{2}, k+\frac{1}{2}}^{n+\frac{1}{2}}+\mu_{x} \nabla_{y}(v \omega)_{j+\frac{1}{2}, k+\frac{1}{2}}^{n+\frac{1}{2}}\right\} .
\end{aligned}
$$

Here, $f_{j, k}^{\prime}$ and $g_{j, k}^{\prime}$, denote discrete 'numerical slopes' in the $x$ - and respectively $y$-directions. Let us consider two examples.

As our first example, we set these numerical slopes to zero, $f^{\prime} \equiv g^{\prime} \equiv$ 0 . Then $\omega^{n+1 / 2}=\bar{\omega}^{n}$ and the central scheme (B.4)-(B.5) is reduced to the classical Lax-Friedrichs (LxF) scheme

$$
\begin{aligned}
\bar{\omega}_{j+\frac{1}{2}, k+\frac{1}{2}}^{n+1}= & \frac{1}{4}\left(\bar{\omega}_{j, k}^{n}+\bar{\omega}_{j+1, k}^{n}+\bar{\omega}_{j, k+1}^{n}+\bar{\omega}_{j+1, k+1}^{n}\right) \\
& -\Delta t\left\{\mu_{y} \nabla_{x}(u \omega)_{j+\frac{1}{2}, k+\frac{1}{2}}^{n}+\mu_{x} \nabla_{y}(v \omega)_{j+\frac{1}{2}, k+\frac{1}{2}}^{n}\right\} .
\end{aligned}
$$


Here is the key observation. By the discrete incompressibility relation (B.3) we find that $\bar{\omega}^{n+1}$ can be rewritten as a convex combination of the neighboring averages at $t=t^{n}$, namely

$$
\begin{aligned}
& \bar{\omega}_{j+\frac{1}{2}, k+\frac{1}{2}}^{n+1}= \frac{1}{4}\left(1+2 \lambda_{x} u_{j, k}^{n}+2 \lambda_{y} v_{j, k}^{n}\right) \bar{\omega}_{j, k}^{n} \\
&+\frac{1}{4}\left(1-2 \lambda_{x} u_{j+1, k}^{n}+2 \lambda_{y} v_{j+1, k}^{n}\right) \bar{\omega}_{j+1, k}^{n} \\
&+\frac{1}{4}\left(1+2 \lambda_{x} u_{j, k+1}^{n}-2 \lambda_{y} v_{j, k+1}^{n}\right) \bar{\omega}_{j, k+1}^{n} \\
&+\frac{1}{4}\left(1-2 \lambda_{x} u_{j+1, k+1}^{n}-2 \lambda_{y} v_{j+1, k+1}^{n}\right) \bar{\omega}_{j+1, k+1}^{n} \\
&=: \sum_{|\alpha-(j+1 / 2)|=1 / 2} \theta_{\alpha, \beta} \bar{\omega}_{\alpha, \beta}^{n}, \quad \sum \theta_{\alpha, \beta}=1 . \\
&|\beta-(k+1 / 2)|=1 / 2
\end{aligned}
$$

Observe, that the $\theta$ 's are nonnegative under the CFL condition $\lambda_{x}|u|+$ $\lambda_{y}|v| \leqslant 1 / 2$. By convexity, therefore,

$$
\phi\left(\bar{\omega}_{j+\frac{1}{2}, k+\frac{1}{2}}^{n+1}\right) \leqslant \sum \theta_{\alpha, \beta} \phi\left(\bar{\omega}_{\alpha, \beta}^{n}\right)
$$

for all convex $\phi$ 's and thanks to the incompressibility relation (B.3) we find that $\omega(\cdot, \cdot, t)$ is a renormalized 'entropy' solution satisfying the discrete analogue of (2.3)

$$
\begin{aligned}
\phi\left(\bar{\omega}_{j+\frac{1}{2}, k+\frac{1}{2}}^{n+1} \leqslant \leqslant\right. & \frac{1}{4}\left\{\phi\left(\bar{\omega}_{j, k}^{n}\right)+\phi\left(\bar{\omega}_{j+1, k}^{n}\right)+\phi\left(\bar{\omega}_{j, k+1}^{n}\right)+\phi\left(\bar{\omega}_{j+1, k+1}^{n}\right)\right\} \\
& -\Delta t\left\{\mu_{y} \nabla_{x}\left(u^{n} \phi\left(\omega^{n}\right)\right)_{j+\frac{1}{2}, k+\frac{1}{2}}\right. \\
& \left.+\mu_{x} \nabla_{y}\left(v^{n} \phi\left(\omega^{n}\right)\right)_{j+\frac{1}{2}, k+\frac{1}{2}}\right\}
\end{aligned}
$$

It follows that the total mass of our piecewise-constant approximate solution, $\int \phi\left(\omega\left(x, y, t^{n}\right)\right.$ is nonincreasing in time.

$$
\int \phi\left(\omega\left(x, y, t^{n}\right)\right) \leqslant \int \phi(\omega(x, y, 0)), \quad \forall \text { convex } \phi .
$$

In particular, $\omega(\cdot, 0) \mapsto \omega\left(\cdot, t^{n}\right)$ maps any Orlicz space into itself.

The LxF scheme (B.6) is a first-order, low-resolution scheme. Higher resolution is obtained if we set the discrete slopes $f^{\prime}, g^{\prime}$ as appropriate numerical derivatives. Namely, second order accuracy is guaranteed wherever these slopes approximate the corresponding derivatives, $f_{j, k}^{\prime} \sim$ $\Delta x \cdot f_{x}\left(x_{j}, y_{k}, t^{n}\right)+\mathcal{O}(\Delta x)^{2}, g_{j, k}^{\prime} \sim \Delta y \cdot g_{y}\left(x_{j}, y_{k}, t^{n}\right)+\mathcal{O}(\Delta y)^{2}$. 
There is a variety of choices to reconstruct such discrete slopes from the given cell-averages. As our second example for the class of central schemes, we set

$$
\begin{gathered}
\omega_{j, k}^{\prime}=M M\left\{\theta\left(\bar{\omega}_{j+1, k}^{n}-\bar{\omega}_{j, k}^{n}\right), \frac{1}{2}\left(\bar{\omega}_{j+1, k}^{n}-\bar{\omega}_{j-1, k}^{n}\right),\right. \\
\left.\theta\left(\bar{\omega}_{j, k}^{n}-\bar{\omega}_{j-1, k}^{n}\right)\right\}, \\
\omega_{j, k}^{\prime}=M M\left\{\theta\left(\bar{\omega}_{j, k+1}^{n}-\bar{\omega}_{j, k}^{n}\right), \frac{1}{2}\left(\bar{\omega}_{j, k+1}^{n}-\bar{\omega}_{j, k-1}^{n}\right),\right. \\
\left.\theta\left(\bar{\omega}_{j, k}^{n}-\bar{\omega}_{j, k-1}^{n}\right)\right\} .
\end{gathered}
$$

Here, $M M$, denotes the usual Min-Mod limiter

$$
M M\left(\left\{w_{k}\right\}\right)= \begin{cases}0, & \text { if }\left\{\exists(i, j) \mid \operatorname{sgn}\left(w_{i}\right) \neq \operatorname{sgn}\left(w_{j}\right)\right\}, \\ s \cdot \min \left|w_{k}\right|, & \text { if }\left\{\forall k^{\prime} s \mid \operatorname{sgn}\left(w_{k}\right)=s\right\},\end{cases}
$$

and $\theta \in(0,2)$, is a free parameter which retains the non-oscillatory properties of the approximate solution. We note in passing that stability enforces this type of nonlinear reconstruction of discrete slopes from the cell-averages of the vorticity. Straightforward divided differences are, of course, another accurate choice but they sacrifice stability. Following the argument along the lines of [27, Theorem 4.1] one finds that under a restricted CFL condition (outlined below), $\bar{\omega}^{n+1}$ can be written as a convex combination of its neighboring gridvalues at $t=t^{n}$. We conclude

THEOREM B.1.-Consider the two-dimensional central scheme (B.4)-(B.5), complemented by the streamfunction computation of the velocity field (B.2), and discrete slopes using the $\theta$-dependent Min-Mod limiter (B.10).

\{i\} Then for any $\theta<2$ there exists a constant,

$$
C_{\theta}=\frac{\sqrt{36+10 \theta(2-\theta)}-6}{20 \theta},
$$

such that if the CFL condition, $\max \left\{\lambda_{x}|u|, \lambda_{y}|v|\right\} \leqslant C_{\theta}$ is fulfilled, then (B.9) holds.

\{ii\} In particular, the evolution mapping $\omega(\cdot, 0) \mapsto \omega\left(\cdot, t^{n}\right)$ maps any Orlicz space into itself, i.e., (2.5) holds.

The last statement of $H_{\text {loc }}^{-1}$-stability implies that the finite difference solution, $\omega^{\Delta t}(\cdot, t)$, is an approximate solution (satisfying properties $\mathcal{P} 1$, 
$\mathcal{P} 2$ and $\left.\mathcal{P} 3^{\prime}\right)$, and $L^{2}$-convergence follows for $\omega^{\Delta t}(\cdot, 0)$ in the host of $2 \mathrm{D}$ rearrangement-invariant spaces outlined in Section 2.

\section{REFERENCES}

[1] Adams R., Sobolev Spaces, Pure and Applied Mathematics, Vol. 65, Academic Press, 1975.

[2] Anderson C., An Introduction to Vortex Methods, Lecture Notes in Math., Vol. 1360, Springer, Berlin, 1968.

[3] Bell J.B., Colella P., Glaz H.M., A Second-order projection method for the incompressible Navier-Stokes equations, JCP 85 (1989) 257-283.

[4] Bennett C., Intermediate spaces and the class $L \log ^{+} L$, Arkiv Mat. 2 (1973) 215228.

[5] Bennett C., Rudnick K., On Lorentz-Zygmund spaces, Dissert. Math. 175 (1980) $1-72$.

[6] Bennett C., Sharpley R., Interpolation of Operators, Pure and Applied Mathematics, Vol. 129, Academic Press, 1988.

[7] Chacon-Rebollo T., Hou T., A Lagrangian finite element method for the 2-D Euler equations, CPAM 43 (1990) 735-767.

[8] Caffarelli L., Kohn R., Nirenberg L., Partial regularity of suitable solutions of the Navier-Stokes equations, CPAM 35 (1982) 771-831.

[9] Chae D., Weak solutions of 2-D Euler equations with initial vorticity in $L(\log L)$, J. Differential Equations 103 (1993) 323-337.

[10] Chae D., Weak solutions of 2-D incompressible Euler equations, Nonlin. Analysis: TMA 23 (1994) 629-638.

[11] Chen G.-Q., The theory of compensated compactness and the system of isentropic gas dynamics, Preprint, MSRI-00527-91, Math. Sci. Res. Inst., Berkeley.

[12] Chorin A., A numerical method for solving incompressible viscous flow problems, JCP 2 (1967) 12-26.

[13] Constantin P., E W., Titi E., Onsager's conjecture on the energy conservation for solutions of Euler's equation, Comm. Math. Phys. 165 (1994) 207-209.

[14] DeVore R., Lucier B., Wavelets, Acta Numerica 1 (1992) 1-56.

[15] DiPerna R., Lions P.-L., Ordinary differential equations Sobolev spaces and transport theory, Invent. Math. 98 (1989) 511-547.

[16] DiPerna R., Majda A., Concentrations in regularizations for 2D incompressible flow, Comm. Pure Appl. Math. 40 (1987) 301-345.

[17] DiPerna R., Majda A., Reduced Hausdorff dimension and concentration-cancelation for 2-D incompressible flow, J. Amer. Math. Soc. 1 (1988) 59-95.

[18] DiPerna R., Majda A., Oscillations and concentrations in weak solutions of the incompressible fluid equations, Comm. Math. Phys. 108 (1987) 667-689.

[19] Delort J.-M., Existence de nappes de tourbillon en dimension deux, J. Amer. Math. Soc. 4 (1991) 553-586.

[20] Donaldson T.K., Trudinger N.S., Orlicz-Sobolev spaces and imbedding theorems, J. Funct. Anal. 8 (1971) 52-75.

[21] E W., Liu J.-G., Finite difference schemes for incompressible flows in the velocityimpulse density formulation, JCP 130 (1997) 67-76. 
[22] Giga Y., Miyakawa T., Navier-Stokes flows in $\mathbb{R}^{3}$ and Morrey spaces, Comm. PDE 14 (1989) 577-618.

[23] Henshaw W., Kreiss H.-O., Reyna L., A forth-order accurate difference approximation for the incompressible Navier-Stokes equations, Comput. Fluids 23 (1994) 575-593.

[24] Hou T.Y., Wetton B.T.R., Second-order convergence of a projection scheme for the incompressible Navier-Stokes equations with boundaries, SINUM 30 (3) (1993) $609-629$.

[25] Hounie J., Lopes Filho M.C., Nussenzveig Lopes H.J., Schochet S., A priori temporal regularity for the streamfunction of $2 \mathrm{D}$ incompressible, inviscid flow, Nonlinear Analysis Theor. 35 (1999) 871-884.

[26] Krasny R., Computing vortex sheet motion, in: Proc. Inter. Congress Math. Vol. I, II, Kyoto 1990, Math. Soc. Japan, 1991, pp. 1573-1583.

[27] Levy D., Tadmor E., Non-oscillatory central schemes for the incompressible 2-D Euler equations, Mathematical Research Letters 4 (1997) 1-20.

[28] Lin F., A new proof of Caffarelli-Kohn-Nirenberg's theorem, Preprint.

[29] Liu J.G., Xin Z., Convergence of vortex methods for weak solutions to the 2D Euler equations with vortex sheet data, CPAM 48 (1995) 611-628.

[30] Lions P.L., Mathematical Topics in Fluid Mechanics, Vol. 1, Incompressible Models, Oxford Lecture Series in Mathematics and its Applications, Vol. 3, Clarendon Press, 1996.

[31] Majda A., Remarks on weak solutions for vortex sheets with a distinguished sign, Ind. Univ. Math. J. 42 (1993) 921-939.

[32] Meyer Y., Wavelets and Operators, Cambridge Studies in Mathematics, Vol. 37, Cambridge Univ. Press, 1992.

[33] Morgulis A.B., On existence of two-dimensional nonstationary flows of an ideal incompressible liquid admitting a curl nonsummable to any power greater than 1, Siberian Math. J. 33 (1992) 934-937.

[34] Murat F., A survey on compensated compactness, in: Cesari L. (Ed.), Contributions to Modern Calculus of Variations, Pitman Research Notes in Mathematics Series, Wiley, New York, 1987, pp. 145-183.

[35] Nussenzveig Lopes H.J., A refined estimate of the size of concentration sets for 2D incompressible inviscid flow, Ind. Univ. Math. J. 46 (1997) 165-182.

[36] Onsager L., Statistical hydrodynamics, Nuovo Cimento (Supplemento) 6 (1949) 279-287.

[37] Scheffer V., An inviscid flow with compact support in space-time, J. Geom. Anal. 3 (1993) 343-401.

[38] Schochet S., The point-vortex method for periodic weak solutions of the 2-D Euler equations, Comm. Pure Appl. Math. 49 (1996) 911-965.

[39] Shnirelman A., On the non-uniqueness of weak solution of the Euler equations, Comm. Pure Appl. Math. 50 (1997) 1261-1286.

[40] Tartar L., Compensated compactness and applications to partial differential equations, in: Nonlinear Analysis and Mechanics, Heriot-Watt Symposium, IV, Pitman, London, 1979.

[41] Temam R., Navier-Stokes Equations, North-Holland, Amsterdam, 1977.

[42] Tian G., Xin Z., Gradient estimation on Navier-Stokes equations, Preprint.

[43] Trudinger N., On imbeddings into Orlicz spaces and some applications, J. Math. and Mechanics 17 (1967) 473-483. 
[44] Vecchi I., Wu S., On $L^{1}$-vorticity for 2-D incompressible flow, Manuscripta Math. 78 (1993) 403-412.

[45] Vishik M., Hydrodynamics in Besov spaces, Anch. Rat. Mech. Anal. 145 (1998) 197-214.

[46] Vishik M., Incompressible flows of an ideal fluid with vorticity in borderline spaces of Besov type, Ann. Sci. Ecole Norm. Sup. 32 (1999) 769-812.

[47] Yudovich V.I., Non-stationary flow of an ideal incompressible liquid, USSR Comp. Math. and Math. Phys. 3 (1963) 1407-1456. English transl.

[48] Yudovich V.I., Uniqueness theorem for the basic nonstationary problem in the dynamics of an ideal incompressible fluid, Math. Res. Letters 2 (1995) 27-38.

[49] Ziemer W.P., Weakly Differentiable Functions, Graduate Texts in Mathematics, Vol. 120, Springer, 1989. 